\title{
Comparative metabolites profiling of Polygonatum cyrtonema Hua seed during sand storage and germination
}

\section{Wenwu Zhang ( $\sim$ 18855175767@163.com )}

Anhui Agricultural University https://orcid.org/0000-0002-9026-3730

Jinfeng Tong

Anhui Agricultural University

Long Xia

Anhui Agricultural University

Xiaoyun Zhu

Anhui Agricultural University

Rui Ran

Anhui Agricultural University

\section{Yan Chang}

Anhui Agricultural University

Jie Zhang

Anhui Agricultural University

\section{Yongping Cai}

Anhui Agricultural University

Qing Jin

Anhui Agricultural University

\section{Research article}

Keywords: cyrtonema Hua, sand storage, seed germination, metabolic profiling, GC-MS

Posted Date: November 10th, 2020

DOl: https://doi.org/10.21203/rs.3.rs-74229/v1

License: (1) (i) This work is licensed under a Creative Commons Attribution 4.0 International License.

Read Full License 


\section{Abstract \\ Background}

The seeds of Polygonatum cyrtonema Hua have dormancy phenomenon. Previous studies have shown that sand storage factors effects of the seed dormancy of $P$. cyrtonema Hua seeds and enhance the seed germination process. Subsequently, metabolic activities and different changes during the sand storage and germination process of $P$. cyrtonema Hua seed has not been heavily researched.

\section{Results}

In this study the changes in the metabolites of $P$. cyrtonema Hua seeds at different sand storage times and germination stages, we used untargeted metabolomics to determine them. Most of the sugar and glycoside contents in seed coat increased after $30 \mathrm{~d}$ on the other hand, in peeled seeds increased at $30 \mathrm{~d}$ and decreased at $60 \mathrm{~d}$ after sand storage treatment. The content of proline and benzoic acid decreased in the seed coat after sand storage. PCA, OPLS-DA and HCA showed that the contents of most metabolites increased after $7 \mathrm{~d}$ and decreased after $14 \mathrm{~d}$ of seed germination. The process of $7 \mathrm{~d}$ to $14 \mathrm{~d}$ was the key stage of seed germination of $P$. cyrtonema Hua. Differential metabolic pathway analysis showed that seed germination was controlled by multiple metabolic pathways. Metabolic correlation revealed the interdependence between seed germination metabolites and metabolic pathways.

\section{Conclusion}

Sand storage can significantly increase the rate of seed germination and play a vital role in seed dormancy of $P$. cyrtonema Hua. There was inherent differences in metabolites during different storage time and germination stages in $P$. cyrtonema Hua. Our work provides a first glimpse of the metabolome in seed germination of $P$. cyrtonema Hua, and provides a valuable informations for revealing the mechanism of breaking seed dormancy.

\section{Background}

P.cyrtonema Hua is a famous medicinal plant,distributed in China. The rhizome of P.cyrtonema Hua has become more popular due to high nutritional and medicinal value, mainly. that are used to treat disorders of the lung, kidney and spleen, and more importantly to prevent aging [1]. The major active ingredients of $P$. cyrtonema Hua are Polygonatum sibiricum polysaccharides [2]. At present, the reproduction of $P$. cyrtonema Hua mainly depends on the rhizome, but long-term asexual reproduction causes germplasm degradation and thereby leads to diseases and insect pests related problems. The $P$. cyrtonema Hua seed reproduction is due to its large number and easy operation in actual production, which are effectively reduce production costs and solve the above problems. However, the low reproduction rate of $P$. cyrtonema Hua seeds in the natural environment has resulted in the need to use a large number of seeds 
in production practices to obtain sufficient seedlings, which results in a waste of resources and inefficiency. The seeds of $P$. cyrtonema Hua show comprehensive dormancy [3] which prevents seeds from germinating under unfavorable physical conditions [4,5]. In many wild species, seeds are dormant at maturity and do not germinate until dormancy release after dispersal, even after exposed to optimal conditions. Germination is a complex trait that is influenced by endogenous and environmental factors $[6,7]$.

There are many methods including chemical, biological, and physical methods to alleviate the seed dormancy. Stratification treatment is commonly used to break seed dormancy. Cold stratification has been widely used for breaking the dormancy of seeds germination in the natural habitat in spring, e.g. Carthamus tinctorius L. [8], Acer morrisonense [9]. Phytohormones are signaling molecules for communication between the three seed compartments (seed embryo, endosperm, and seed coat), and play key roles in coordinating appropriate seed formation [10]. Low temperature sand storage is optimal for break the seed dormancy of $P$. cyrtonema Hua [11]. Nevertheless, in some other plants, room temperature sand storage is more effective [12]. Among them, sand storage (low temperature stratification treatment ) is the most common method to end the seed dormancy. In the dormancybreaking treatment, seeds are treated with wet sand storage at $4{ }^{\circ} \mathrm{C}$. Few studies on the mechanism of breaking dormancy and changes of metabolites during dormancy breaking are reported.

To reveal the mechanism of breaking dormancy, many studies have focused on different physiological aspects in the process of dormancy. Metabolomics has also been applied to analyze the changes in metabolites of breaking seed dormancy. For example, a gas chromatography-mass spectrometer (GCMS) has been used to reveal the metabolite's changes of Safron corm from dormancy release to germination [13]. Takahashi analyzed the poor germination of Gentiana triflora seed by using targeted metabonomics [14]. Recently, metabolomic characterization based on the non-targeted GC-MS of seed germination has been reported $[15,16]$.In present study, the seeds of $P$. cyrtonema Hua were used as raw materials. GC-MS technique was used to study the changes of metabolites in the process of sand storage and seed germination.

\section{Results}

\section{Effects of sand storage on the seed germinatio n rate of $P$. cyrtonema Hua}

The seeds of $P$. cyrtonema Hua treated with sand storage at $4{ }^{\circ} \mathrm{C}$ in winter for $30 \mathrm{~d}$. (Fig.1). The rate of seeds germination was significantly higher (3.69 times) after the treatment with sand storage as compared to non-sand storage (control). . These results showed that treatment of sand storage plays an important role in the germination of $P$. cyrtonema Hua seeds.

\section{Metabolic profiling of the seed coat and peeled seeds of $P$. cyrtonema Hua at different storage time}

The metabolic profiles of the seed coat and peeled seeds during four sand storage stages were investigated using GC-MS. Metabolome-wide expression profiling showed highest metabolite expression 
during the treatment of sand storage. Through comparative analysis, 85 and 74 metabolites were identified in seed coat and peeled seeds, respectively (Fig.3). In the metabolite profiles were significant differences existed between the seed coat and peeled seeds. There are 65 metabolites detected in both the seed coat and peeled seeds, but 20 metabolites were only detected in the seed coat and 9 metabolites were only detected in peeled seeds (Fig.3B). The specific metabolites of seed coat included four carbohydrates, six alcohols, four organic acids, four nitrogenous compounds, and two other compounds, as well as, the nine metabolites specific to peeled seeds included two carbohydrates, five organic acids, and two other metabolites were presented (Fig.3A).

\section{Changes of metabolites in seed coat and peeled seeds of $P$. cyrtonema Hua in metabolic pathways during different sand storage time}

Our study sought to obtain a more detailed overview of the abundance of the identified substances and, similarities and differences in each storage time of both organs (Fig.4). In starch and sucrose metabolism, trehalose showed a similar trend in seed coat and peeled seeds during sand storage. Trehalose increased at $30 \mathrm{~d}$ of sand storage, decreased significantly at $60 \mathrm{~d}$, and slightly increased at 90 d. The sucrose, fructose, and maltose in peeled seeds were significantly higher than those in other periods when stored in the sand for $30 \mathrm{~d}$. The sucrose and maltose of seed coat decreased significantly after $60 \mathrm{~d}$ of sand storage. In the TCA cycle, seed coat and peeled seeds had the same metabolites, but the changing trend of metabolites was different in seed coat and peeled seeds. During sand storage, the content of pyruvate, succinate, and malate in peeled seeds showed similar trends, and the metabolites of sand storage at $60 \mathrm{~d}$ were significantly higher than those in the other three periods, indicating that the TCA cycle was relatively active at this time. Succinate of seed coat showed a significant downward trend in the sand storage process and increased slightly at $90 \mathrm{~d}$. Pyruvate of seed coat was significantly higher than that in the other three periods during $30 \mathrm{~d}$ of sand storage. Malate of seed coat and peeled seeds changed in opposite directions.

These results indicating that, most of the sugar and glycosides content in the seed coat increased at $30 \mathrm{~d}$, indicating that the macromolecular nutrients in the seed coat began to decompose into soluble sugar, and the other sand storage time changed differently. Most of the sugars and glycosides in peeled seeds increased at $30 \mathrm{~d}$ of sand storage and decreased at $60 \mathrm{~d}$ of sand storage, which indicated that the macromolecular nutrients in seeds began to decompose into soluble sugars in preparation for seeds to break dormancy. After $60 \mathrm{~d}$ of sand storage, sugar and glycosides began to degrade, indicating that the seeds have been in a relatively active state, began to consume sugar and glycosides to prepare for seed germination. The changing trend of amino acids in seed coat and peeled seeds were also different. The content of most amino acids in seed coat decreased with the extension of sand storage time, and most of the amino acids in peeled seeds were the highest at $60 \mathrm{~d}$ of sand storage. Proline was an important product of dormancy release [20]. The content of proline in the seed coat decreased after sand storage, while the content of proline in peeled seeds increased after $60 \mathrm{~d}$ of sand storage. Benzoic acid is only detected in the seed coat, and benzoic acid is considered to be an endogenous inhibitor [21]. 


\section{Morphological changes of $P$. cyrtonema Hua seeds at different germination stages}

Through the dynamic observation of the germination process (Fig.5), it was found that the seeds of $P$. cyrtonema Hua started to germinate on $14 \mathrm{~d}$, and the seeds began to show small buds, and the corms started outgrowth after the germination of $21 \mathrm{~d}$. Then the corm began to germinate and grow to form radicle and hypocotyl.

\section{Analysis of metabolites during seed germination of $P$. cyrtonema Hua based on GC-MS}

GC-MS was used to analyze the metabolites during the germination process of $P$. cyrtonema Hua. A total of 96 metabolites were isolated and identified, including 22 amino acids, 20 sugars and glycosides, 22 organic acids, 13 alcohols and esters, 8 nitrogen compounds, 4 fatty acids, and 7 other compounds.

Firstly, the samples were analyzed by using PCA to detect the distribution of metabolites at different germination stages of $P$. cyrtonema Hua seeds (Fig.6). The first principal component covered $22.3 \%$, and the second principal component covered $30.9 \%$. From the dispersion trend of the score map samples, it can be seen that the seed germination $0 \mathrm{~d}$ can be obviously distinguished from other germination periods, $21 \mathrm{~d}, 28 \mathrm{~d}, 35 \mathrm{~d}$ cannot be distinguished obviously.

The OPLS-DA, a supervised pattern recognition method, was further employed to identify the metabolites during the germination of $P$. cyrtonema Hua seeds (Fig.7, VIP>1.0, $p<0.05$ ). There are 17, 27, 9, 7, 1 metabolites that showed significantly changed and greatly contributed to typing from $0 \mathrm{~d}$ vs. $7 \mathrm{~d}, 7 \mathrm{~d}$ vs. 14 d, 14 d vs. 21 d, 21 d vs. 28 d, 28 d vs. 35 d, respectively(Additional file1: Table S1).

The dynamic changes of metabolites during the seed germination stages were analyzed by HCA. As shown in Fig.8, the metabolites during seed germination can be divided into three clusters. Cluster I mainly consists of two organic acids (succinic acid and 2, 4, 6-trihydroxybenzoic acid), three amino acids (glycine, 4-aminobutyric acid, $\beta$-alanine), and a glycoside compound. The contents of these metabolites decreased gradually during the seed germination process. Cluster II mainly includes 9 amino acids (proline, alanine, glutamic acid, etc.), 10 organic acids (citric acid, mandelic acid, glyceric acid, etc.), 7 sugars and glycosides (maltose, trehalose, moschose, etc.), 4 alcohols (inositol, glycerol, squalinositol, etc.), one fatty acid, four nitrogen-containing compounds, and four other metabolites after seed germination for $7 d$, these metabolites contents firstly increase, and most of them decreased after $14 d$ of germination. Cluster III mainly consists of 12 sugars and glycosides (sucrose, cellobiose, fructose, etc.), 10 amino acids (tryptophan, L-lysine, tyrosine, etc.), 11 organic acids (malic acid, lactic acid, niacin, etc.), 9 alcohols and esters, 5 nitrogen compounds, 3 fatty acids (linoleic acid, stearic acid, palmitic acid) and 4 other compounds. The changing trend of cluster III was partly similar to that of cluster II. These metabolite contents gradually increased at $14 \mathrm{~d}$ of seed germination, and decreased at $21 \mathrm{~d}$. However, compared with cluster I, the change was not significant. In summary, from the results of thermographic analysis of metabolites, it was found that the content of most compounds began to increase at $7 \mathrm{~d}$ of germination, and to decrease at $14 \mathrm{~d}$. 


\section{Changes of metabolites during seeds germinationof $P$. cyrtonema Hua}

Through the analysis of KEGG pathway of differential metabolites, the differential metabolites were enriched to 32, 32, 4, and 9 pathways from 0 d vs. 7 d, 7 d vs. 14 d, 14 d vs. 21 d, 21 d vs. $28 d$, respectively. There was only one differential metabolite in $28 \mathrm{~d}$ vs. $35 \mathrm{~d}$, and on the other side, there was no differential metabolic pathway (Additional file2: Table S2). The significantly enriched metabolic pathway was mainly related to amino acid metabolism, sugar metabolism, inositol phosphate metabolism and citric acid cycle. The metabolic pathways with significant enrichment were citric acid cycle, arginine and proline metabolism, starch and sucrose metabolism, etc. (Additional file2: Table S2).

A pair-wise comparison between all the stages was performed. We observed significant metabolite changes $(p<0.05)$ between $0 \mathrm{~d}$ and $7 \mathrm{~d}$ as depicted on the metabolic map (Fig.9A). In $7 \mathrm{~d} \otimes$ we observed significant increase in sucrose (2.02), sorbitol (1.75), glycine (1.28), serine (1.85), phenylalanine (1.97), carbamate (2.10), citric acid (1.70), malic acid (1.57), glutamic acid (6.27), proline (1.38), aspartic acid (2.05), asparagine (2.66), palmitic acid (1.89) and stearic acid (2.63) , accompanied by a significant decrease in inositol (0.14), tryptophan (0.59) and succinic acid $(0.46)$

We also observed significant metabolite changes $(p<0.05)$ between stages $7 \mathrm{~d}$ and $14 \mathrm{~d}$ (Fig.9B). In $14 \mathrm{~d}$, our study showed significant increase in sucrose (1.27), glucose (1.90), carbamate (1.14), valine (1.33), isoleucine (1.36), proline (1.38), glycine (1.28), serine (1.27), malic acid (1.30), L-hydroxyproline (1.34), phenylalanine (1.97), palmitic acid (1.30) and ethanolamine (1.24) accompanied by significant decrease in inositol (0.90), tryptophan (0.9) ,Glycolic acid (0.76).

When the seeds germinated at $21 \mathrm{~d}$, the differential metabolities contents (mainly were amino acids and inositol) were decreased. On the $28 \mathrm{~d}$ of germination, the content of most of the differential metabolism decreased significantly, except for isoleucine and 4-hydroxypyridine, the content of other differential metabolites decreased significantly, including sugar, niacin and sorbitol. In summary, it can be concluded that the seed germination of $P$. cyrtonema Hua may be affected by a variety of metabolic pathways.

\section{Correlation analysis of metabolites during seed germination of $P$. cyrtonema Hua}

In this study, the changes of metabolic network among metabolites $(r \geq 0.8, p<0.001)$ during the germination of $P$. cyrtonema Hua were analyzed. As shown in Fig.10, a total of 250 pairs of metabolites were significantly correlated, and all of them were positively correlated. It mainly existed between amino acids, organic acids, sugars, glycosides and alcohols, accounting for $48 \%$ of the total correlation, such as proline and aspartic acid $(r=0.87, p=5.41 \mathrm{E}-12)$, maltose $(r=0.963, p=5.45 \mathrm{E}-21)$, inositol $(r=0.843, p=$ 1.08E-10), phosphate $(r=0.914, p=6.89 \mathrm{E}-15)$. Glutamine and lysine $(r=0.836, p=2.24 \mathrm{E}-10)$. In addition, malic acid and serine $(r=0.82, p=9.79 \mathrm{E}-10)$, sucrose $(r=0.819, p=9.91 \mathrm{E}-10)$. Succinic acid and glycine $(r=0.928, p=4.05 \mathrm{E}-16), \gamma$-aminobutyric acid $(r=0.875, p=3.12 \mathrm{E}-12), \beta$-alanine $(r=0.91, p=1.52 \mathrm{E}-14), 2$ O-glycerol-a-D-galactose $(r=0.894, p=2.08 \mathrm{E}-13)$. It shows that the metabolic pathways of amino acids, sugars and organic acids act together during seed germination $₫$ Fig. $10 \rrbracket$. 


\section{Discussion}

Compared with the control group, the sand storage treatment can significantly improve the seeds germination rate of $P$. cyrtonema Hua. This is consistent with the research of Bian, $\mathrm{F}$ [22], used of sand storage to release dormancy and improve the germination rate of Taxus yunnanensis. During the postripening development of seeds in the process of sand storage, the internal macromolecular substances and enzyme activities were in a certain dynamic change. In the post-ripening stage, the macromolecular substances (such as starch, etc.) can be decomposed into soluble sugars, such as glucose, sucrose, fructose, etc., and then participate in seed germination and basic physiological respiration needs. During the storage at room temperature, the soluble sugar in the seeds increased at first and then decreased with the extension of storage time. It is speculated that the starch decomposition in the seeds at the early stage of storage may provide soluble sugar, and with the extension of storage time, the respiration may become weaker, resulting in a decrease during the utilization of soluble sugar [23]. Soluble sugars include most monosaccharides and oligosaccharides, which can be used as mediators for energy storage and transfer in plants, as well as ligands for structural substances and functional molecules such as glycoproteins. From the analysis of the changes of metabolites in the seeds of $P$. cyrtonema Hua treated with low temperature sand storage at different times, it can be seen that the contents of most sugar and glycosides in the peeled seeds started to increase gradually from $0 \mathrm{~d}$ to $60 \mathrm{~d}$ of sand storage times, and decreased significantly at $90 \mathrm{~d}$. After $60 \mathrm{~d}$, the sugar and glycosides (glucose, sucrose, fructose) began to degrade, indicating that the seed has been in a relatively active state, started to consume carbohydrates to prepare for seed germination, which is consistent with the research of $M a, L$, etc [24].

Since the seed was imbibed, its internal metabolism was activated to provide nutrition and energy for seed germination. Through the dynamic observation of the germination process of $P$. cyrtonema Hua seeds (Fig. 5), it was found that the seeds began to germinate at $14 \mathrm{~d}$, and the seeds started to show small buds, and the corms appeared at $21 \mathrm{~d}$ after germination. At the later stage, the corm began to germinate and grow to form radicle and hypocotyl. Sugar, as an important source of carbon source in seeds, provides conditions for seed germination [25]. In this study, during seed germination for 0 to $14 \mathrm{~d}$, the content of disaccharide (trehalose, maltose, sucrose, etc.) began to increase, and these metabolites decreased after $14 \mathrm{~d}$ of germination. These disaccharides are soluble sugars and are considered to be rapidly available [26]. The generation of energy may be related to the cell growth in the seed, including the expansion of the protocambium ring and the radicle [27]. After $14 \mathrm{~d}$ of seed germination, the content of disaccharide decreased, which indicated that the glycolysis pathway and tricarboxylic acid cycle were affected in these stages. At the same time, we also found that the content of intermediate metabolites (malic acid, succinic acid, citric acid) of tricarboxylic acid cycle decreased at the stage of $14 \mathrm{~d}$ to $28 \mathrm{~d}$ of germination. During seed germination process, seeds take energy through other metabolic pathways, such as fermentation and amino acid consumption [28]. Amino acid metabolism is essential during seed germination because it must be consistent with the changes of carbohydrates and carbohydrates in seeds in order to drive cell metabolism and promote seed germination [29]. The changing trend of most amino acids in seeds was the same as that of disaccharide. It has been found that $\gamma$-aminobutyric acid is related to the production of seed energy [30]. In the process of seed germination, the content of $\mathrm{Y}^{-}$ 
aminobutyric acid was the highest after $0 \mathrm{~d}$ of germination, and began to decrease in other stages. Besides, inositol is associated with many aspects of plant physiology, such as carbohydrate metabolism, seed germination, stress response and cell wall formation, as well as cell division [31,32]. Our study showed that the content of inositol in seeds increased at the stage of $0 \mathrm{~d}$ to $7 \mathrm{~d}$ of germination, and decreased at the beginning of germination $(14 \mathrm{~d})$, these indicated that the inositol in seeds was used for seed germination.

\section{Conclusion}

These results indicated that the germination and dormancy process was significantly increased after the treatments of sand storage in $P$. cyrtonema Hua seeds. During the sand storage process, the metabolites in $P$. cyrtonema Hua seeds and peeled seeds change during different sand storage times. In this process of seed sand storage from $0 \mathrm{~d}$ to $90 \mathrm{~d}, 10$ kinds of sugar and glycosides content in the seed coat continued to increase, 7 kinds of them continuously decreased, and another 5 kinds of them increased first and then decreased, the highest content was at $30 \mathrm{~d}$. The content of most carbohydrates and glycosides in the peeled seeds increased at $30 \mathrm{~d}$ after the treatment of sand storage, and gradually decreased after $60 \mathrm{~d}$. The content of most amino acids in the seed coat gradually decreased with the extension of the sand storage times, while the content of most amino acids in the peeled seeds increased first and then decreased, the highest content was at $60 \mathrm{~d}$. We found that the content of proline,an important product of releasing dormancy $\square$ decreased in the seed coat after sand storage. Benzoic acid, an endogenous inhibitors, decreased also.

During the germination of $P$. cyrtonema Hua seeds, from $0 \mathrm{~d}$ to $35 \mathrm{~d}$, the content of most amino acids, organic acids, sugars, and alcohols showed a trend of first increasing and then decreasing, as well as, the content reached the maximum value from $7 \mathrm{~d}$ to $14 \mathrm{~d}$. A variety of differential metabolites were screened from the germination process of $P$. cyrtonema Hua seeds, which were mainly concentrated in the first 14 days of germination. According to the metabolic map, the germination of $P$. cyrtonema Hua seeds is affected by various metabolic pathways. Among them, the metabolic pathways of amino acids, sugars and organic acids showed a positive correlation.

\section{Methods}

\section{Plant material}

The P. cyrtonema Hua seeds were provided by the Polygonatum planting base of Sansanfulin Chinese Medicinal Materials Co., Ltd.,„China in September 2017, which located in Zongwen Village, Ducun Township, Qingyang County, Chizhou City, Anhui Province in China (30³2' 30.8"N 117²43'54.8"E), and authenticated by Professor Yongping Cai (School of Life Sciences, Anhui Agricultural University). Sand storage and seed germination of $P$. cyrtonema Hua were carried out in the laboratory of Anhui Agricultural University. 
The effect of sand storage on the seed germination rate of P. cyrtonema Hua

After rubbing, rinsing, washing and drying the fresh $P$. cyrtonema Hua fruit, sand storage treatment was carried out at $4{ }^{\circ} \mathrm{C}$ in winter, and water was sprayed regularly to maintain humidity. The seeds of $P$. cyrtonema Hua were taken out, planted in the hole seedling tray, and cultured in a constant-temperature incubator at $26^{\circ} \mathrm{C}$. For this, one hundred seeds per treatment were used in triplicates, and count the seed germination rate after 30 days. Using seeds not in sand storage as the control. SPSS19 software was used for data statistical analysis. The germination rate was calculated using the following formula:

Germination rate $=$ a total number of germinated seeds $/$ total number of tested seeds $* 100 \%$.

Changes of metabolites of P. cyrtonema Hua seed coat and peeled seeds in different sand storage times

The seeds of $P$. cyrtonema Hua were collected on these stages $0 \mathrm{~d}, 30 \mathrm{~d}, 60 \mathrm{~d}$, and $90 \mathrm{~d}$ in sand storage, respectively. The seed coat and peeled seeds(including seed embryo and endosperm)were separated and frozen with liquid nitrogen and stored in a refrigerator at $-80^{\circ} \mathrm{C}$ until used for GC-MS to identify metabolites.

Changes of metabolites during the germination of P. cyrtonema Hua seeds

The seeds of $P$. cyrtonema Hua after 60 days of sand storage were soaked in $\mathrm{GA}_{3}$ for $24 \mathrm{~h}$, and the seeds germinated for $0 \mathrm{~d}, 7 \mathrm{~d}, 14 \mathrm{~d}, 21 \mathrm{~d}, 28 \mathrm{~d}$ and $35 \mathrm{~d}$, respectively. They are frozen with liquid nitrogen and stored in a refrigerator at $-80^{\circ} \mathrm{C}$ until used for GC-MS to identify metabolites.

\section{Extraction of Metabolites of P. cyrtonema Hua seeds}

The protocol for GC-MS metabolite extraction was following that of the previously described procedure [13]. The collected samples $(100 \mathrm{mg})$ were grinded in a mortar using liquid nitrogen, then transferred into $10 \mathrm{~mL}$ centrifuge tubes. After adding $1.4 \mathrm{~mL}$ of $100 \%$ methanol (precooled at $-20{ }^{\circ} \mathrm{C}$ ) and vortexing for $30 \mathrm{~s}, 60 \mu \mathrm{L}$ of polar internal standard $\left(0.2 \mathrm{mg} \cdot \mathrm{mL}^{-1}\right.$ 1,2-benzenediol in methanol) was added to the tube. The samples were vortexed again for $30 \mathrm{~s}$ and placed into an ultrasound machine for $30 \mathrm{~min}$ at $40{ }^{\circ} \mathrm{C}$, followed by centrifugation for $20 \mathrm{~min}$ at $4000 \mathrm{rpm}$. Then, we transferred the supernatant to a $10 \mathrm{~mL}$ centrifuge tube which was followed by the addition of $750 \mu \mathrm{L}$ of chloroform and $1.4 \mathrm{~mL}$ of $\mathrm{ddH}_{2} \mathrm{O}$ and the mixture was vortexed for $30 \mathrm{~s}$ before centrifugation at $4000 \mathrm{rpm}$ for $20 \mathrm{~min}$. The supernatant was transferred into a new tube and blow-dried with nitrogen. Subsequently, $60 \mu \mathrm{L}$ of methoxypyridine (20 $\mathrm{mg} \cdot \mathrm{mL}^{-1}$ ) was added and vortexed for $30 \mathrm{~s}$. After a reaction time of $2 \mathrm{~h}$ at $37^{\circ} \mathrm{C}, 60 \mu \mathrm{L}$ of $\mathrm{N}, \mathrm{O}-$ bis(trimethylsilyl) trifluoroacetamide (BSTFA) reagent (containing $1 \%$ trimethylchlorosilane) was added to the tube. After reacting for $1.5 \mathrm{~h}$ at $37^{\circ} \mathrm{C}$, the samples were used for GC-MS analysis.

\section{Detection conditions by GC-MS}

The GC-MS data were obtained using an Agilent 7000B (Agilent, Santa Clara, CA, USA) system equipped with an DB-5 MS capillary column $(60 \mathrm{~m} \times 0.25 \mathrm{~mm}, 0.25 \mu \mathrm{m}$ film thickness, Agilent J \& W Scientific, 
Folsom, CA, USA); The injection inlet temperature was $280^{\circ} \mathrm{C}$, source temperature of $250^{\circ} \mathrm{C}$, an inlet temperature of $250^{\circ} \mathrm{C}$. Split ratio $10: 1$ and injection volume was $1 \mu \mathrm{L}$ The following temperature program: initial temperature of $40^{\circ} \mathrm{C}$ and held for $5 \mathrm{~min}$, increased by $8^{\circ} \mathrm{C} / \mathrm{min}$ to $280^{\circ} \mathrm{C}$, and held for $5 \mathrm{~min}$ with a constant flow of $1 \mathrm{~mL} / \mathrm{min}$ helium as the carrier gas. The solvent delay time was $14 \mathrm{~min}$, and $\mathrm{m} / \mathrm{z}$ range was 33-600. The detection was by an Agilent 5977C Triple Quad with the following settings: electrospray ionization (ESI) source $(70 \mathrm{eV})$; source temperature of $230^{\circ} \mathrm{C}$, the quad temperature of $150{ }^{\circ} \mathrm{C}$ and $\mathrm{m} / \mathrm{z}$ range was $35-780$.

\section{Data Analysis}

To ensure the stability of the assay, we performed six biological replications and analyzed them under identical conditions. The QCs were injected at regular intervals throughout the GC-MS analytical run to provide a set of data from which repeatability could be assessed. All the GC-MS data were processed by XCMS running under the $\mathrm{R}$ package, which produced a matrix of features with the associated retention times, accurate masses, and peak areas. All internal peaks were removed from the data set. The resulting data were normalized to the total peak area of each sample in Excel 2010. Principal component analysis (PCA) and orthogonal partial least squares-discriminant analysis (OPLS-DA) models were generated using Simca-P software version 14.0. The differential metabolites were selected based on the combination of the statistically significant threshold of variable influence on projection (VIP) values obtained from the OPLS-DA model and the p-value from a two-tailed Student's t-test of the normalized peak area; metabolites with VIP $>1.0$ and $p<0.05$, respectively, were selected. For GC-MS analysis, metabolites were identified by searching the commercial database NIST11 [17] after their mass spectra were deconvoluted by the Automated Mass Spectral Deconvolution and Identification System (AMDIS) [18]. Peaks with a similarity index of more than $70 \%$ were tentatively identified as metabolites. The identified metabolites were mapped to general biochemical pathways according to their annotation in the Kyoto Encyclopedia of Genes and Genomes (KEGG) database. Pathway analysis was performed using MetaboAnalyst 4.0 [19]. The metabolome data of the differential metabolites were used for different analysis and subsequently normalized by sum, log-transformed, and Pareto-scaled thereby compared with the KEGG pathway library of Arabidopsis thaliana. The heatmap was generated with R software.

\section{Abbreviations}

$\mathrm{GA}_{3}$ Gibberellic

PCA Principal component analysis

OPLS-DA Orthogonal partial least squares discriminant analysis

VIP Variable importance in the projection

HCA Hierarchical cluster analysis

QC Quality Control 


\section{Declarations}

\section{Ethics approval and consent to participate}

Not applicable.

\section{Consent for publication}

Not applicable.

\section{Availability of data and materials}

All data generated or analysed during this study are included in this published article and its supplementary information files.

\section{Competing interests}

The authors declare that they have no competing interests.

\section{Funding}

This work was supported by the major project of Anhui Department of Education (03087060) 『and the Industry-University-Research Cooperation Project of Chizhou City, Anhui Province(11006232).

\section{Author contributions}

Q.J. and Y.P.C. conceived and designed the experiments; J.T.『W.Z., L.X., X.Z., R.R., J.Z., and Y.C. performed the experiments; J.T., W.Z. analyzed the data and wrote the paper; Q.J., and Y.P.C. revised the paper; All authors read and approved the final manuscript.

\section{Acknowledgments}

We thank Qingyang for the experimental materials.

\section{References}

1. Chinese Pharmacopoeia Commission. Pharmacopoeia of the People's Republic of China vol.1. Beijing:China Medical Science Press, 2015, 306-307.

2. Zeng, G.F.; Zhang, Z.Y.; Lu, L.; Xiao, D.Q.; Xiong, C.X.; Zhao, Y.X.; Zong, S.H. Protective effects of Polygonatum sibiricum polysaccharide on ovariectomyinduced bone loss in rats. Ethnopharmacole, 2011, 136(1), 224-229.

3. Zhang, Y.J.; Zhang, Y.C.; Li, Y.G.; Liu, F.; Guo, H.P. Seed Dormant Characteristics of Polygonatum sibiricum Red. Bulletin of Botanical Research, 2010, 30(6), 753-757. 
4. Baskin, C.C.; Baskin, J.M. Seeds: ecology, biogeography, and evolution of dormancy and germination. ElsevierAcademic Press, 2014.

5. Chen, S.Y.; Chien, C.T.; Baskin, J.M.; Baskin, C.C. Storage behavior and changes in concentrations of abscisic acid and gibberellins during dormancy break and germination in seeds of Phellodendron amurense var. wilsonii (Rutaceae). Tree physiology, 2009, 30, 275-284.

6. Joosen, R.V.L,; Arends, D.; Li, Y.; Willems, L.A.J; Keurentjes, J.J.B.; Ligterink, W.; Jansen, R.; Hilhorst, H.W.M. Identifying genotype-by-environment interactions in the metabolism of germinating Arabidopsis seeds using Generalized Genetical Genomics. Plant Physiology, 2013, 162, 553-566.

7. Shu, K.; Liu, X.D.; Xie, Q.; He, Z.H. Two faces of one seed: hormonal regulation of dormancy and germination. Molecular Plant, 2016, 9, 34-45.

8. Horikoshi, M.; Sekozawa, Y.; Kobayashi, M.; Saito, K.; Kusano, M.; Sugaya, S. Metabolomics analysis of 'Housui' Japanese pear flower buds during endodormancy reveals metabolic suppression by thermal fluctuation[J]. Plant Physiology and Biochemistry, 2018, 028.

9. Chen, -Y.; Chou, S.H.; Tsai, C.C.; Hsu, W.Y.; Baskin, C.C.; Baskin, J.M.; Chien, C.T.; Kuo-Huang, L.L. Effects of moist cold stratification on germination, plant growth regulators, metabolites and embryo ultrastructure in seeds of Acer morrisonense (Sapindaceae)[J]. Plant Physiol Biochem, 2015, 94:165173.

10. Han, C.X.; Zhen, S.M.; Zhu, G.R.; Bian, Y.W.; Yan, Y.M. Comparative metabolome analysis of wheat embryo and endosperm reveals the dynamic changes of metabolites during seed germination[J]. Plant Physiology and Biochemistry, 2017, S0981942817301328.

11. Zhao, Z.; Pang, Y.X.; Yuan, Y.; Fu, M.X.; Liu, S.Q.; Cao, D.T.; Xian, F.R. Study on the Seed Reproduces Technology of Medicinal Crop Rhizoma Polygonati[J]. Seed, 2005.24(3):11-13

12. Bian, F.; Su, J.; Liu, W.; Li, S.F. Dormancy release and germination of Taxus yunnanensis seeds during wet sand storage. Scientific Reports, 2018, 8(1), 3205.

13. Bagri, J.; Yadav,A.; Anwar, K.; Dkhar, J.; Singla-Pareek, S.L.; Pareek, A. Metabolic shift in sugars and amino acids regulates sprouting in Saffron corm[J]. Scientific Reports, 2017, 7(1):11904.

14. Takahashi, H.; Fujita, K.; Yoshida, C.; Nishihara, M. Metabolite profiling reveals the involvement of aberrant metabolic changes in Gentiana triflora seed showing poor germination[J]. Journal of Pomology and Horticultural Science, 2016, 91(2):8.

15. Kazmi, R.H.; Willems, L.A.J.; Joosen, R.V.L.; Khan, N.; Ligterink, W.; Hilhorst, H.W.M. Metabolomic analysis of tomato seed germination. Metabolomics 2017, 13, (12).

16. Silva, A.T.; Ligterink, W.; Hilhorst, H.W.M. Metabolite profiling and associated gene expression reveal two metabolic shifts during the seed-to-seedling transition in Arabidopsis thaliana. Plant Molecular Biology 2017, 95, (4-5), 481-496.

17. Vinaixa, M.; Schymanski, E.L.; Neumann, S.; Navarro, M.; Salek, R.M.; Yanes, O. Mass spectral databases for LC/MS- and GC/MS-based metabolomics: State of the field and future prospects. Trac-Trend Anal Chem 2016, 78, 23-35. 
18. Meyer, M.R.; Peters, F.T.; Maurer, H.H. Automated Mass Spectral Deconvolution and Identification System for GC-MS Screening for Drugs, Poisons, and Metabolites in Urine. Clinical Chemistry 2010, $56,(4), 575-584$.

19. Xia, J.; Sinelnikov, I.V.; Han, B.; Wishart, D.S. MetaboAnalyst 3.0-making metabolomics more meaningful. Nucleic Acids Research 2015, 43, (W1), W251-W257.

20. He, Y.; Gan, S. A novel zinc-finger protein with a proline-rich domain mediates ABA-regulated seed dormancy in Arabidopsis[J]. Plant Molecular Biology, 2004, 54(1):1-9.

21. Leone, C.M.; Celletti, C.; Gaudiano, G.; Puglisi, P.A.; Fasolino, A.; Cruccu, G.; Camerota, F.; Truini, A. Pain due to Ehlers-Danlos Syndrome Is Associated with Deficit of the Endogenous Pain Inhibitory Control. Pain medicine (Malden, Mass.)

22. Bian, F.; Su, J.; Liu, W.; Li, S. J. S. r., Dormancy release and germination of Taxus yunnanensis seeds during wet sand storage. 2018, 8, (1), 3205.

23. .Dirk, L.M.A; van der Krol, A.R; Vreugdenhil, D; Hilhors, H.W.M;; Bewley, J.D. Galactomannan, soluble sugar and starch mobilization following germination of Trigonella foenum-graecum seeds[J]. Plant Physiology and Biochemistry (Paris), 1999, 37(1):41-50.

24. Ma, L.; Ling, W.; Shengchao, Y.; Tao, L. Study on the Physiological and Biochemical Changes of Paris polyphylla Smith var. yunnanensis. Seeds during the Mature Period [J]. Journal of Yunnan Agricultural University (Natural Science) 2015, V30(005):766-770.

25. Eveland, A.L.; Jackson, D.P. Sugars, signalling, and plant development. Exp. Bot. 2012, 63, (9), 33673377.

26. Sami, F.; Yusuf, M.; Faizan, M.; Faraz, A.; Hayat, S. Role of sugars under abiotic stress. Plant Physiology and Biochemistry 2016, 109, 54-61.

27. Mazlan, O.; Aizat, W.M.; Baharum, S.N.; Azizan, K.A.; Noor, N.M. Metabolomics analysis of developing Garcinia mangostana seed reveals modulated levels of sugars, organic acids and phenylpropanoid compounds[J]. Scientia Horticulturae, 2018, 233: 323-330.

28. Rosental, L.; Nonogaki, H.; Fait, A. Activation and regulation of primary metabolism during seed germination. Seed Science Research 2014, 24, (1), 1-15.

29. Araujo, W.L.; Tohge, T.; Ishizaki, K.; Leaver, C.J.; Fernie, A.R. Protein degradation-an alternative respiratory substrate for stressed plants. Trends in Plant Science 2011, 16, (9), 489-498.

30. Shelp, B.J.; Walton, C.S.; Snedden, W.A.; Tuin, L.G.; Oresnik, I.J.; Layzell, D.B. GABA shunt in developing soybean seeds is associated with hypoxia[J]. Physiologia Plantarum, 1995, 94(2): 219228.

31. Loewus F.A.; Loewus M.W. Myo-inositol: its biosynthesis and metabolism[J]. Annual Review of Plant Physiology, 1983, 34(1): 137-161.

32. Loewus F.A.; Murthy P.P.N. Myo-Inositol metabolism in plants[J]. Plant Science, 2000, 150(1): 1-19.

\section{Additional Files}


Additional file1: Table S1 Differential metabolites during seed germination of $P$. cyrtonema Hua 


\begin{tabular}{|c|c|c|c|c|}
\hline Number & Speculate compound & $\mathrm{p}$ & VIP & Fold \\
\hline \multicolumn{5}{|c|}{$0 \mathrm{~d}$ vs. $7 \mathrm{~d}$} \\
\hline 1 & Succinic acid & $\begin{array}{l}2.81 \mathrm{E}- \\
02\end{array}$ & 4.78345 & 0.46 \\
\hline 2 & Sorbitol & $\begin{array}{l}1.44 \mathrm{E}- \\
03\end{array}$ & 4.67522 & 1.75 \\
\hline 3 & Serine & $\begin{array}{l}1.81 \mathrm{E}- \\
02\end{array}$ & 3.00181 & 1.85 \\
\hline 4 & Malic acid & $\begin{array}{l}2.26 \mathrm{E}- \\
02\end{array}$ & 2.94297 & 1.57 \\
\hline 5 & Carbodiimide & $\begin{array}{l}3.35 \mathrm{E}- \\
02\end{array}$ & 2.81324 & 1.64 \\
\hline 6 & Ethylamine & $\begin{array}{l}2.54 \mathrm{E}- \\
02\end{array}$ & 2.46337 & 1.71 \\
\hline 7 & Myo-Inositol & $\begin{array}{l}2.42 \mathrm{E}- \\
02\end{array}$ & 2.40903 & 0.14 \\
\hline 8 & Hexadecanoic acid & $\begin{array}{l}2.76 \mathrm{E}- \\
02\end{array}$ & 2.40328 & 1.89 \\
\hline 9 & Carbamate & $\begin{array}{l}1.07 \mathrm{E}- \\
02\end{array}$ & 2.38291 & 2.10 \\
\hline 10 & Stearate & $\begin{array}{l}8.26 \mathrm{E}- \\
03\end{array}$ & 2.38062 & 2.63 \\
\hline 11 & D-Psicose & $\begin{array}{l}3.05 \mathrm{E}- \\
02\end{array}$ & 2.36222 & 1.52 \\
\hline 12 & L-Aspartic acid & $\begin{array}{l}2.10 \mathrm{E}- \\
03\end{array}$ & 2.36007 & 2.05 \\
\hline 13 & Sucrose & $\begin{array}{l}8.25 \mathrm{E}- \\
05\end{array}$ & 2.15298 & 2.02 \\
\hline 14 & Glutamine & $\begin{array}{l}4.24 \mathrm{E}- \\
02\end{array}$ & 1.45533 & 6.27 \\
\hline 15 & Citric acid & $\begin{array}{l}4.76 \mathrm{E}- \\
02\end{array}$ & 1.41701 & 1.70 \\
\hline 16 & Asparagine & $\begin{array}{l}3.92 \mathrm{E}- \\
02\end{array}$ & 1.31053 & 2.66 \\
\hline 17 & 2,2'-Bipyridine & $\begin{array}{l}6.89 \mathrm{E}- \\
03\end{array}$ & 1.19252 & 2.00 \\
\hline \multicolumn{5}{|c|}{$7 \mathrm{~d}$ vs. $14 \mathrm{~d}$} \\
\hline 1 & Ethylamine & $5.97 \mathrm{E}-$ & 2.12314 & 0.82 \\
\hline
\end{tabular}




\begin{tabular}{|c|c|c|c|c|}
\hline & & 03 & & \\
\hline 2 & ( \pm )-a-Amino-y-butyrolactone & $\begin{array}{l}4.22 \mathrm{E}- \\
02\end{array}$ & 2.74654 & 0.94 \\
\hline 3 & Glycolic acid & $\begin{array}{l}1.38 \mathrm{E}- \\
02\end{array}$ & 3.7908 & 0.76 \\
\hline 4 & Carbamate & $\begin{array}{l}5.18 \mathrm{E}- \\
03\end{array}$ & 1.07271 & 1.14 \\
\hline 5 & 4-Hydroxypyridine & $\begin{array}{l}3.05 \mathrm{E}- \\
03\end{array}$ & 3.41759 & 1.26 \\
\hline 6 & Ethanolamine & $\begin{array}{l}1.61 \mathrm{E}- \\
02\end{array}$ & 1.13441 & 1.24 \\
\hline 7 & Valine & $\begin{array}{l}4.02 \mathrm{E}- \\
04\end{array}$ & 2.96288 & 1.33 \\
\hline 8 & Dodecane, 4,6-dimethyl- & $\begin{array}{l}1.04 \mathrm{E}- \\
02\end{array}$ & 2.27309 & 2.58 \\
\hline 9 & Isoleucine & $\begin{array}{l}1.26 \mathrm{E}- \\
03\end{array}$ & 2.82617 & 1.36 \\
\hline 10 & D-Proline & $\begin{array}{l}5.33 \mathrm{E}- \\
04\end{array}$ & 2.5892 & 1.38 \\
\hline 11 & Glycine & $\begin{array}{l}1.24 \mathrm{E}- \\
03\end{array}$ & 1.91787 & 1.28 \\
\hline 12 & Serine & $\begin{array}{l}6.53 \mathrm{E}- \\
04\end{array}$ & 2.46712 & 1.27 \\
\hline 13 & Sebacic acid & $\begin{array}{l}5.67 \mathrm{E}- \\
03\end{array}$ & 1.29923 & 1.45 \\
\hline 14 & D-Erythrose & $\begin{array}{l}1.16 \mathrm{E}- \\
02\end{array}$ & 2.25028 & 0.05 \\
\hline 15 & 2,2'-Bipyridine & $\begin{array}{l}7.21 \mathrm{E}- \\
04\end{array}$ & 1.52434 & 1.69 \\
\hline 16 & Malic acid & $\begin{array}{l}5.15 \mathrm{E}- \\
05\end{array}$ & 3.64339 & 1.30 \\
\hline 17 & 4-hydroxyproline & $\begin{array}{l}4.04 \mathrm{E}- \\
05\end{array}$ & 4.23518 & 1.34 \\
\hline 18 & Phenylalanine & $\begin{array}{l}1.42 \mathrm{E}- \\
04\end{array}$ & 1.78747 & 1.94 \\
\hline 19 & 2-(phosphonooxy)acrylate & $\begin{array}{l}1.35 \mathrm{E}- \\
02\end{array}$ & 1.0015 & 0.79 \\
\hline 20 & Sorbitol & $\begin{array}{l}4.40 \mathrm{E}- \\
02\end{array}$ & 1.96719 & 1.46 \\
\hline
\end{tabular}




\begin{tabular}{|c|c|c|c|c|}
\hline 21 & D-(+)-Glucose & $\begin{array}{l}2.12 \mathrm{E}- \\
05\end{array}$ & 5.0083 & 1.90 \\
\hline 22 & $\begin{array}{l}\text { Butylphosphonic acid, heptyl 4-(2-phenylprop-2- } \\
\text { yl)phenyl ester }\end{array}$ & $\begin{array}{l}1.96 \mathrm{E}- \\
04\end{array}$ & 2.2737 & 1.69 \\
\hline 23 & Hexadecanoic acid & $\begin{array}{l}8.56 \mathrm{E}- \\
03\end{array}$ & 2.08823 & 1.30 \\
\hline 24 & Myo-Inositol & $\begin{array}{l}3.75 \mathrm{E}- \\
03\end{array}$ & 1.89028 & 0.90 \\
\hline 25 & Tryptophan & $\begin{array}{l}4.44 \mathrm{E}- \\
02\end{array}$ & 2.09388 & 0.59 \\
\hline 26 & Stearate & $\begin{array}{l}1.49 \mathrm{E}- \\
02\end{array}$ & 1.55603 & 1.28 \\
\hline 27 & Sucrose & $\begin{array}{l}1.40 \mathrm{E}- \\
04\end{array}$ & 2.06912 & 1.27 \\
\hline \multicolumn{5}{|c|}{$14 \mathrm{~d}$ vs. 21} \\
\hline 1 & Borate & $\begin{array}{l}4.81 \mathrm{E}- \\
02\end{array}$ & 1.91705 & 0.60 \\
\hline 2 & Valine & $\begin{array}{l}6.54 \mathrm{E}- \\
03\end{array}$ & 3.41948 & 0.60 \\
\hline 3 & Isoleucine & $\begin{array}{l}2.13 \mathrm{E}- \\
03\end{array}$ & 3.51385 & 0.54 \\
\hline 4 & Pipecolic acid & $\begin{array}{l}1.06 \mathrm{E}- \\
02\end{array}$ & 2.74019 & 0.56 \\
\hline 5 & L-threonine & $\begin{array}{l}3.88 \mathrm{E}- \\
02\end{array}$ & 1.64722 & 0.66 \\
\hline 6 & L-Aspartic acid & $\begin{array}{l}1.76 \mathrm{E}- \\
02\end{array}$ & 1.91418 & 0.65 \\
\hline 7 & Phenylalanine & $\begin{array}{l}3.37 \mathrm{E}- \\
02\end{array}$ & 1.49433 & 0.55 \\
\hline 8 & 2-(phosphonooxy)acrylate & $\begin{array}{l}2.62 \mathrm{E}- \\
03\end{array}$ & 1.14158 & 0.55 \\
\hline 9 & Myo-Inositol & $\begin{array}{l}2.24 \mathrm{E}- \\
02\end{array}$ & 2.43433 & 0.69 \\
\hline \multicolumn{5}{|c|}{$\begin{array}{l}21 d \text { vs. } 28 \\
\text { d }\end{array}$} \\
\hline 1 & 4-Hydroxypyridine & $\begin{array}{l}4.71 \mathrm{E}- \\
02\end{array}$ & 5.73455 & 1.18 \\
\hline 2 & Isoleucine & $\begin{array}{l}1.91 \mathrm{E}- \\
02\end{array}$ & 4.08881 & 1.46 \\
\hline
\end{tabular}




\begin{tabular}{|lllll|}
\hline 3 & Nicotinic acid & $3.54 \mathrm{E}-$ & 1.19889 & 0.60 \\
\hline 4 & Sorbitol & 02 & & \\
\hline 5 & & $2.08 \mathrm{E}-$ & 4.45181 & 0.60 \\
& D-Psicose & 03 & & \\
\hline 6 & & $7.98 \mathrm{E}-$ & 2.85881 & 0.59 \\
& D-Fructose & 03 & & \\
\hline 7 & & $3.31 \mathrm{E}-$ & 3.08833 & 0.56 \\
\hline $28 \mathrm{~d}$ vs. 35 & & 02 & & \\
\hline $\mathrm{d}$ & & $1.32 \mathrm{E}-$ & 6.42316 & 0.44 \\
\hline 1 & & 02 & & \\
\hline & L-rhamnose & & & \\
\hline
\end{tabular}

Additional file2: Table S2 Differential metabolic pathways during seed germination of $P$. cyrtonema Hua 


\begin{tabular}{|c|c|c|}
\hline Number & Metabolic pathway & $p$ \\
\hline \multicolumn{3}{|c|}{$0 \mathrm{~d} v \mathrm{vs} .7 \mathrm{~d}$} \\
\hline 1 & Alanine, aspartate and glutamate metabolism & 2.27E-08 \\
\hline 2 & Tyrosine metabolism & $2.98 \mathrm{E}-08$ \\
\hline 3 & Propanoate metabolism & $2.25 \mathrm{E}-07$ \\
\hline 4 & Butanoate metabolism & $2.25 \mathrm{E}-07$ \\
\hline 5 & Citrate cycle (TCA cycle) & 3.01E-06 \\
\hline 6 & Glyoxylate and dicarboxylate metabolism & $3.40 \mathrm{E}-05$ \\
\hline 7 & Inositol phosphate metabolism & $3.40 \mathrm{E}-05$ \\
\hline 8 & Ascorbate and aldarate metabolism & $3.40 \mathrm{E}-05$ \\
\hline 9 & Lysine biosynthesis & $8.15 \mathrm{E}-05$ \\
\hline 10 & beta-Alanine metabolism & 8.72E-05 \\
\hline 11 & Carbon fixation in photosynthetic organisms & 0.00011777 \\
\hline 12 & Nicotinate and nicotinamide metabolism & 0.00018829 \\
\hline 13 & Arginine and proline metabolism & 0.00018829 \\
\hline 14 & Nitrogen metabolism & 0.00057046 \\
\hline 15 & Aminoacyl-tRNA biosynthesis & 0.0012403 \\
\hline 16 & Glycine, serine and threonine metabolism & 0.0042089 \\
\hline 17 & Cysteine and methionine metabolism & 0.0047203 \\
\hline 18 & Cyanoamino acid metabolism & 0.007338 \\
\hline 19 & Purine metabolism & 0.01063 \\
\hline 20 & Pyrimidine metabolism & 0.01063 \\
\hline 21 & Methane metabolism & 0.012616 \\
\hline 22 & Sphingolipid metabolism & 0.012616 \\
\hline 23 & Sulfur metabolism & 0.022549 \\
\hline 24 & Fructose and mannose metabolism & 0.022549 \\
\hline 25 & Fatty acid biosynthesis & 0.022549 \\
\hline \multicolumn{3}{|c|}{$7 \mathrm{~d}$ vs. $14 \mathrm{~d}$} \\
\hline 1 & Starch and sucrose metabolism & 2.27E-08 \\
\hline
\end{tabular}




\begin{tabular}{|c|c|c|}
\hline 2 & Galactose metabolism & $2.98 \mathrm{E}-08$ \\
\hline 3 & Inositol phosphate metabolism & $2.25 \mathrm{E}-07$ \\
\hline 4 & Ascorbate and aldarate metabolism & $2.25 \mathrm{E}-07$ \\
\hline 5 & Aminoacyl-tRNA biosynthesis & 3.01E-06 \\
\hline 6 & Phenylalanine metabolism & 3.40E-05 \\
\hline 7 & Phenylpropanoid biosynthesis & $3.40 \mathrm{E}-05$ \\
\hline 8 & Tropane, piperidine and pyridine alkaloid biosynthesis & $3.40 \mathrm{E}-05$ \\
\hline 9 & Nitrogen metabolism & 8.15E-05 \\
\hline 10 & Pantothenate and CoA biosynthesis & 8.72E-05 \\
\hline 11 & Glucosinolate biosynthesis & 0.00011777 \\
\hline 12 & Valine, leucine and isoleucine biosynthesis & 0.00018829 \\
\hline 13 & Valine, leucine and isoleucine degradation & 0.00018829 \\
\hline 14 & Glyoxylate and dicarboxylate metabolism & 0.00057046 \\
\hline 15 & Arginine and proline metabolism & 0.0012403 \\
\hline 16 & Phenylalanine, tyrosine and tryptophan biosynthesis & 0.0042089 \\
\hline 17 & Glycine, serine and threonine metabolism & 0.0047203 \\
\hline 18 & Glutathione metabolism & 0.007338 \\
\hline 19 & Tryptophan metabolism & 0.01063 \\
\hline 20 & Indole alkaloid biosynthesis & 0.01063 \\
\hline 21 & Methane metabolism & 0.012616 \\
\hline 22 & Cyanoamino acid metabolism & 0.012616 \\
\hline 23 & Cysteine and methionine metabolism & 0.022549 \\
\hline 24 & Sphingolipid metabolism & 0.022549 \\
\hline 25 & Sulfur metabolism & 0.022549 \\
\hline \multicolumn{3}{|c|}{$14 \mathrm{~d}$ vs. $21 \mathrm{~d}$} \\
\hline 1 & Valine, leucine and isoleucine degradation & 0.0075594 \\
\hline 2 & Glucosinolate biosynthesis & 0.015123 \\
\hline 3 & Valine, leucine and isoleucine biosynthesis & 0.01803 \\
\hline 4 & Aminoacyl-tRNA biosynthesis & 0.031306 \\
\hline
\end{tabular}




\begin{tabular}{|lll|}
\hline $21 \mathrm{~d}$ vs. $28 \mathrm{~d}$ & & \\
\hline 1 & Valine, leucine and isoleucine biosynthesis & $9.02 \mathrm{E}-06$ \\
\hline 2 & Valine, leucine and isoleucine degradation & $9.02 \mathrm{E}-06$ \\
\hline 4 & Glucosinolate biosynthesis & $9.02 \mathrm{E}-06$ \\
\hline 5 & Aminoacyl-tRNA biosynthesis & $9.02 \mathrm{E}-06$ \\
\hline 6 & Fructose and mannose metabolism & $3.80 \mathrm{E}-05$ \\
\hline 7 & Galactose metabolism & $3.80 \mathrm{E}-05$ \\
\hline 8 & Starch and sucrose metabolism & 0.00093938 \\
\hline 9 & Amino sugar and nucleotide sugar metabolism & 0.00093938 \\
\hline
\end{tabular}

Additional file 3: Figure S1 Overlays of TICs by GC-MS analysis of (A) seed coat and (B) peeled seeds under different sand storage time.

Additional file 4: Figure S2 Overlays of total ion chromatograms $\triangle T$ ICs $₫$ by GC-MS analysis in the germinating seeds of $P$. cyrtonema Hua

\section{Figures}

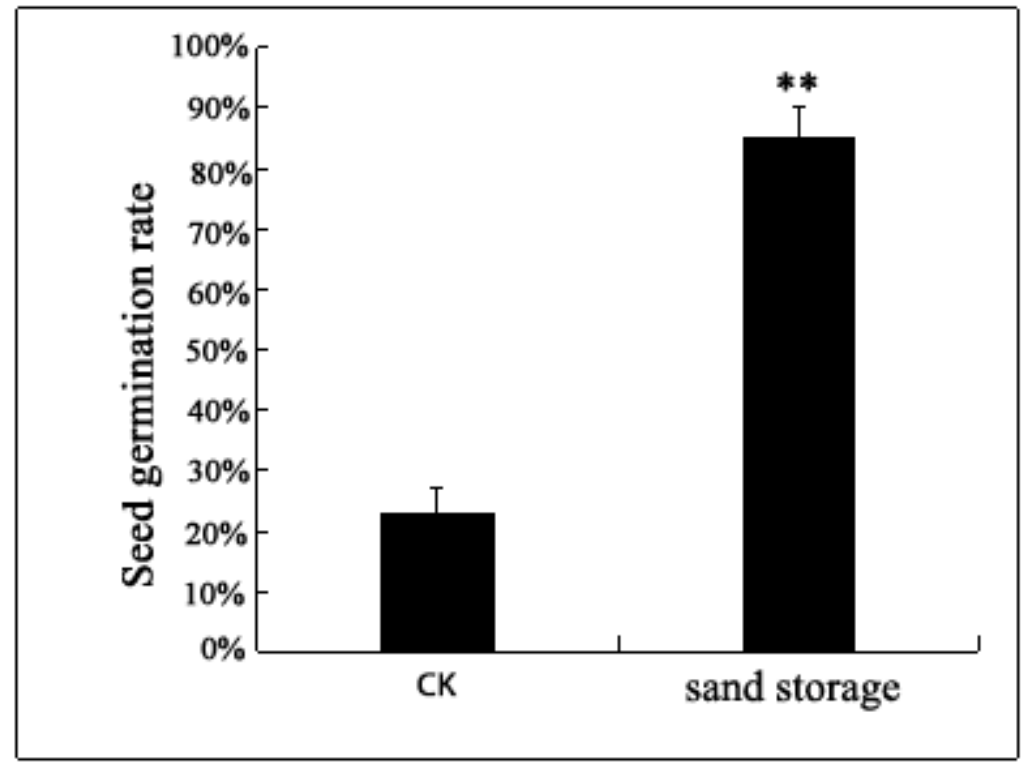

Figure 1

Effects of sand storage on seed germination of P. cyrtonema Hua, extremely significant ${ }^{*} p<0.01$. "CK" in the figure means non sand storage. 


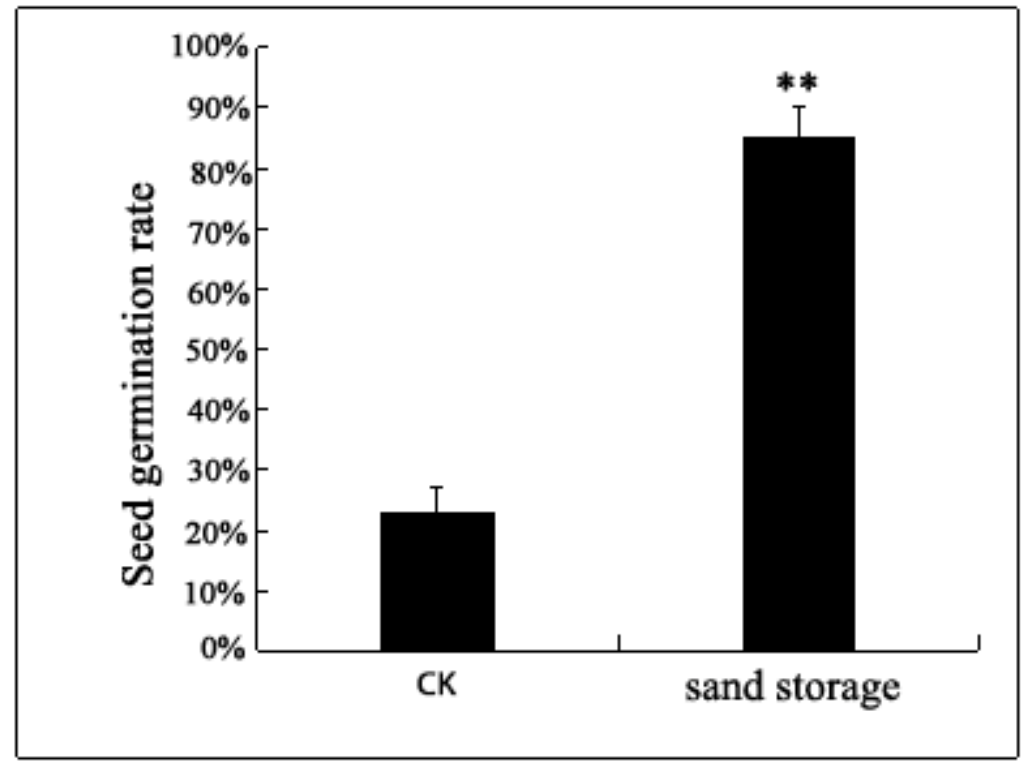

Figure 1

Effects of sand storage on seed germination of P. cyrtonema Hua, extremely significant ${ }^{*} p<0.01$. "CK" in the figure means non sand storage.
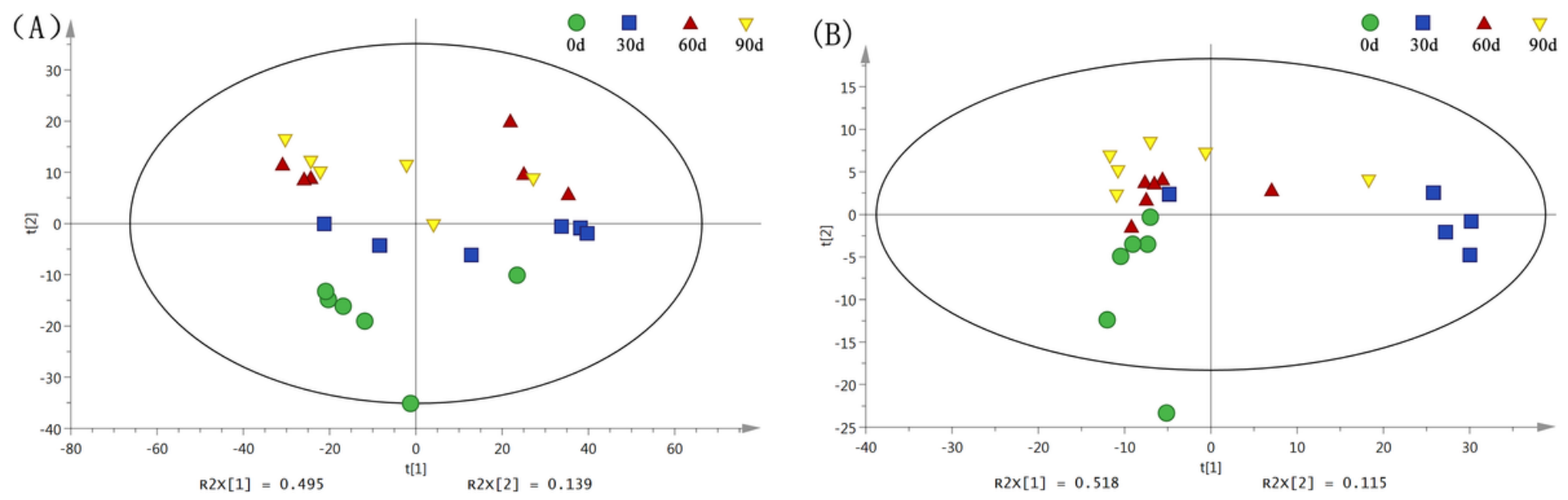

Figure 2

PCA score plots of the metabolites from (A) the seed coat and (B) peeled seeds with four sand storage periods. Periods include $0 \mathrm{~d}$ (non-sand storage), $30 \mathrm{~d}, 60 \mathrm{~d}$, and $90 \mathrm{~d}$. 

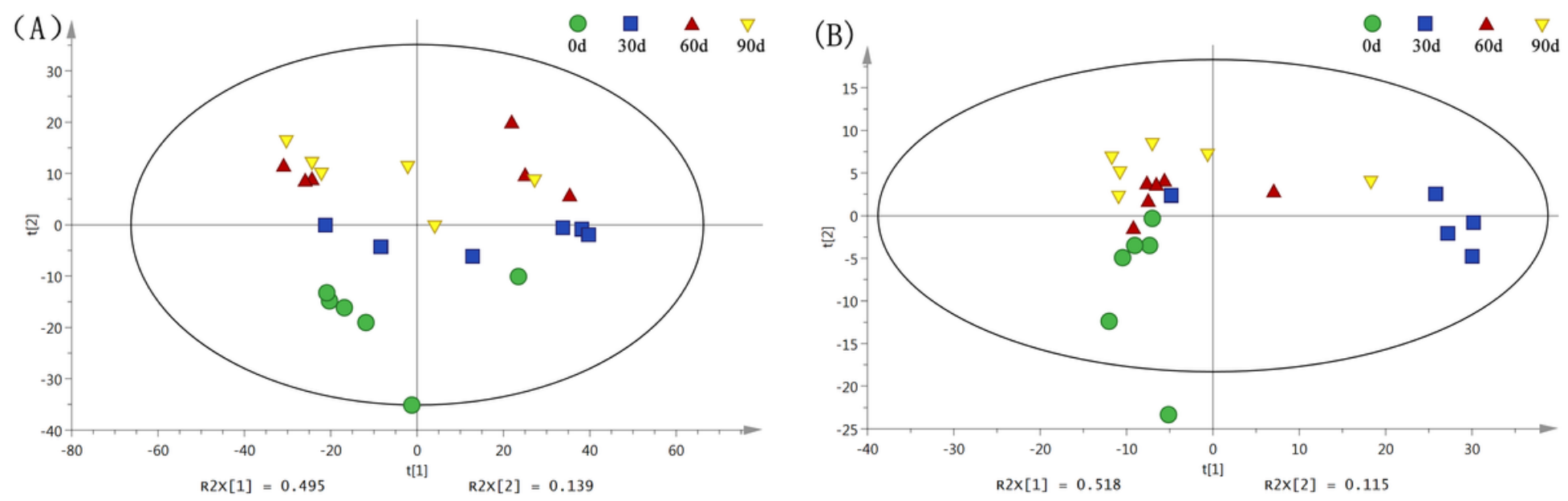

Figure 2

PCA score plots of the metabolites from (A) the seed coat and (B) peeled seeds with four sand storage periods. Periods include $0 \mathrm{~d}$ (non-sand storage), $30 \mathrm{~d}, 60 \mathrm{~d}$, and $90 \mathrm{~d}$.
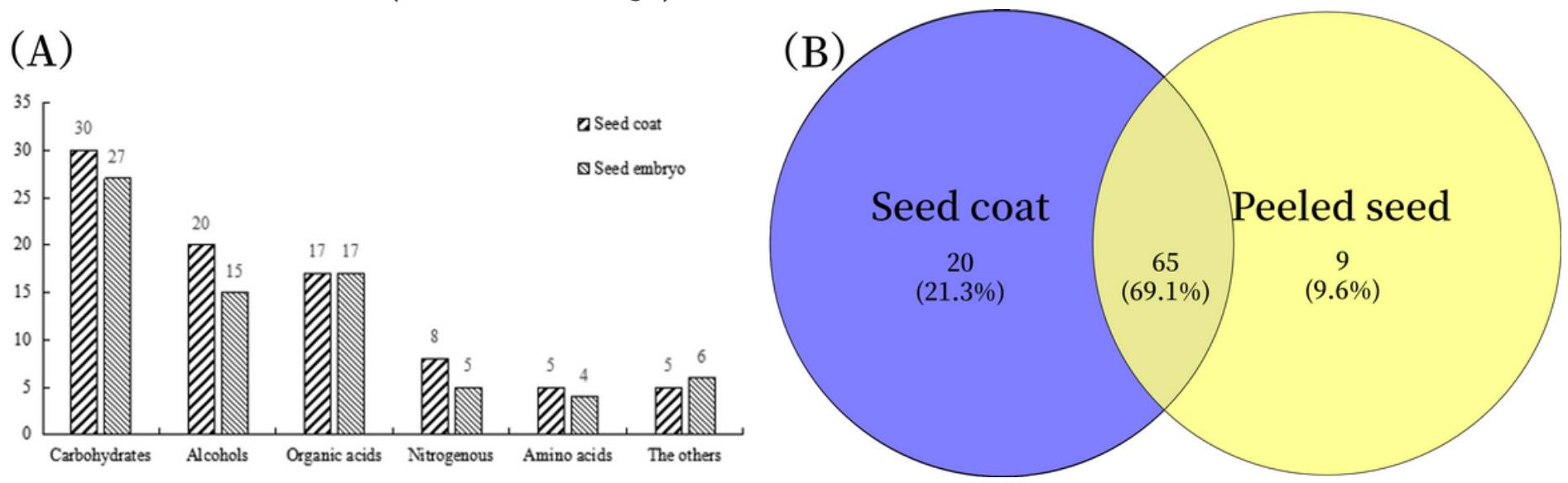

Figure 3

Distribution of the identified metabolites in the storage seed. (A) corresponded to the functional classification of significantly different metabolites in the seed coat and peeled seeds. (B) corresponded to Venn diagram showing the distribution of the significantly different metabolites in the seed coat and peeled seeds.
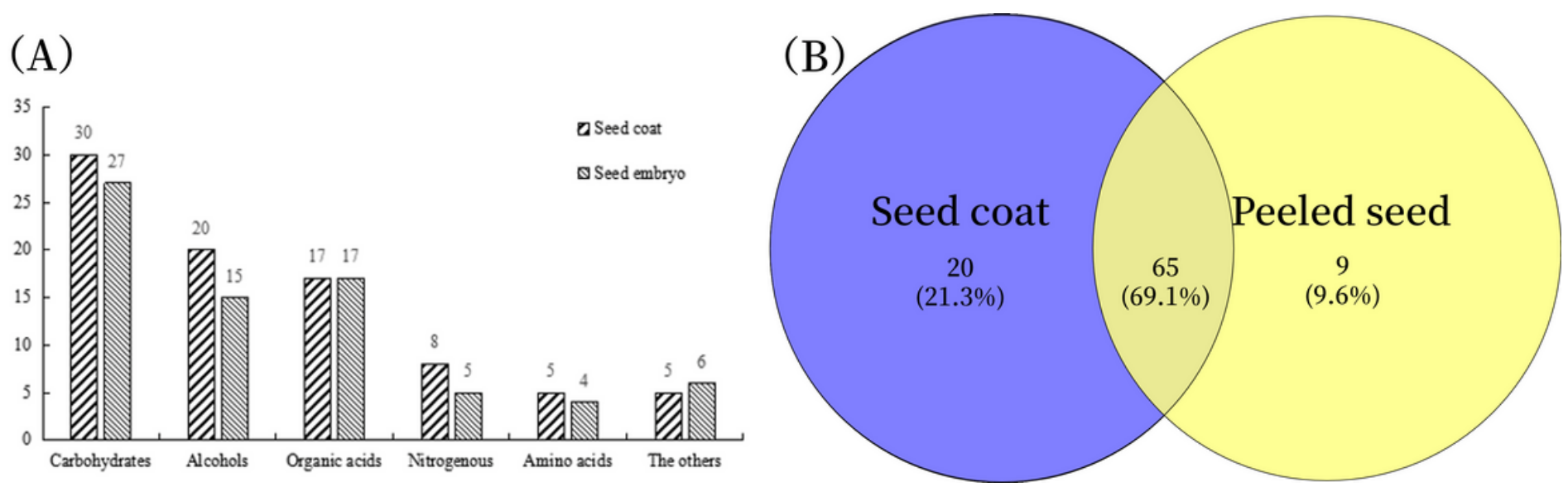


\section{Figure 3}

Distribution of the identified metabolites in the storage seed. (A) corresponded to the functional classification of significantly different metabolites in the seed coat and peeled seeds. (B) corresponded to Venn diagram showing the distribution of the significantly different metabolites in the seed coat and peeled seeds.

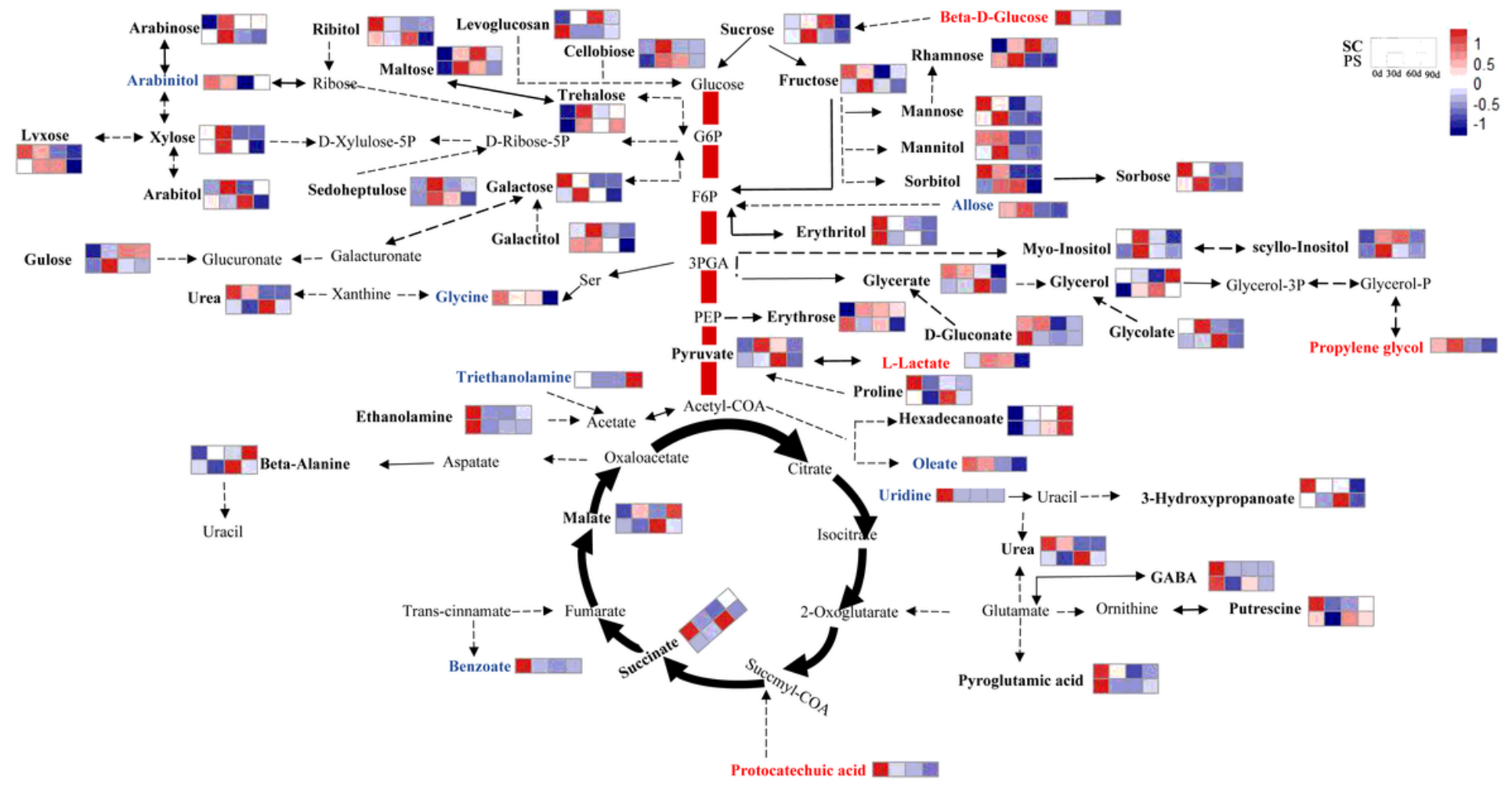

\section{Figure 4}

Primary metabolism pathways of measured metabolites in the seed coat (SC) and peeled seeds (PS). Note: Represented pathways are simplified versions of the TCA, glycolysis, amino acid synthesis, and sucrose synthesis. Within each box, rows represent organs (upper row: SC; lower row: PS). Each column is a sand storage stage (from left to right: $0 \mathrm{~d} ; 30 \mathrm{~d} ; 60 \mathrm{~d} ; 90 \mathrm{~d}$ ), as shown in the upper right corner. Average metabolite intensity is color-coded according to the scale in the upper right corner. Metabolites measured in both seed coat and peeled seeds, non-measured, only measured in the seed coat, only measured in peeled seeds are displayed in black thickening, gray, blue and red font respectively. 


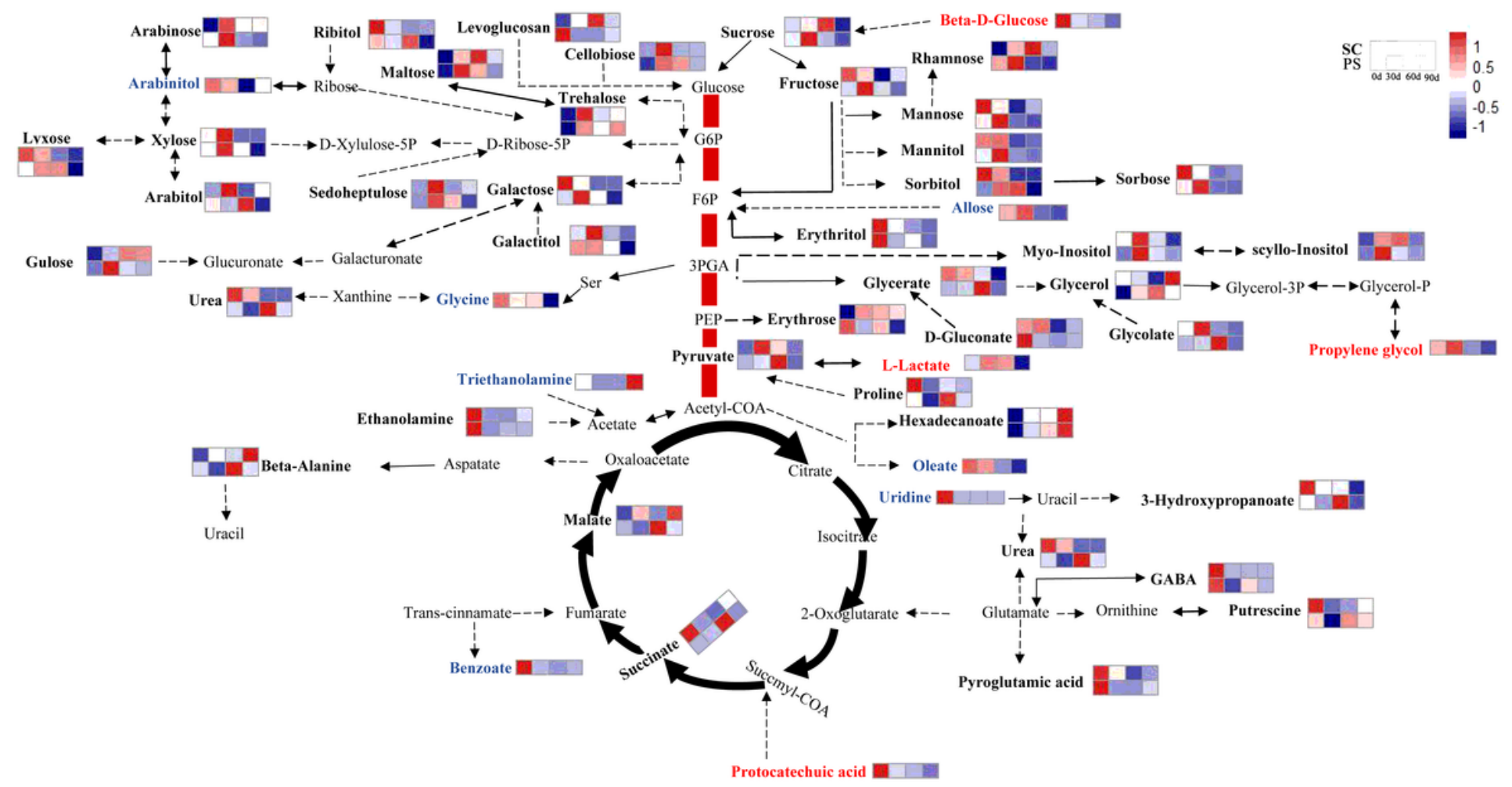

Figure 4

Primary metabolism pathways of measured metabolites in the seed coat (SC) and peeled seeds (PS). Note: Represented pathways are simplified versions of the TCA, glycolysis, amino acid synthesis, and sucrose synthesis. Within each box, rows represent organs (upper row: SC; lower row: PS). Each column is a sand storage stage (from left to right: $0 \mathrm{~d} ; 30 \mathrm{~d} ; 60 \mathrm{~d} ; 90 \mathrm{~d}$ ), as shown in the upper right corner. Average metabolite intensity is color-coded according to the scale in the upper right corner. Metabolites measured in both seed coat and peeled seeds, non-measured, only measured in the seed coat, only measured in peeled seeds are displayed in black thickening, gray, blue and red font respectively. 


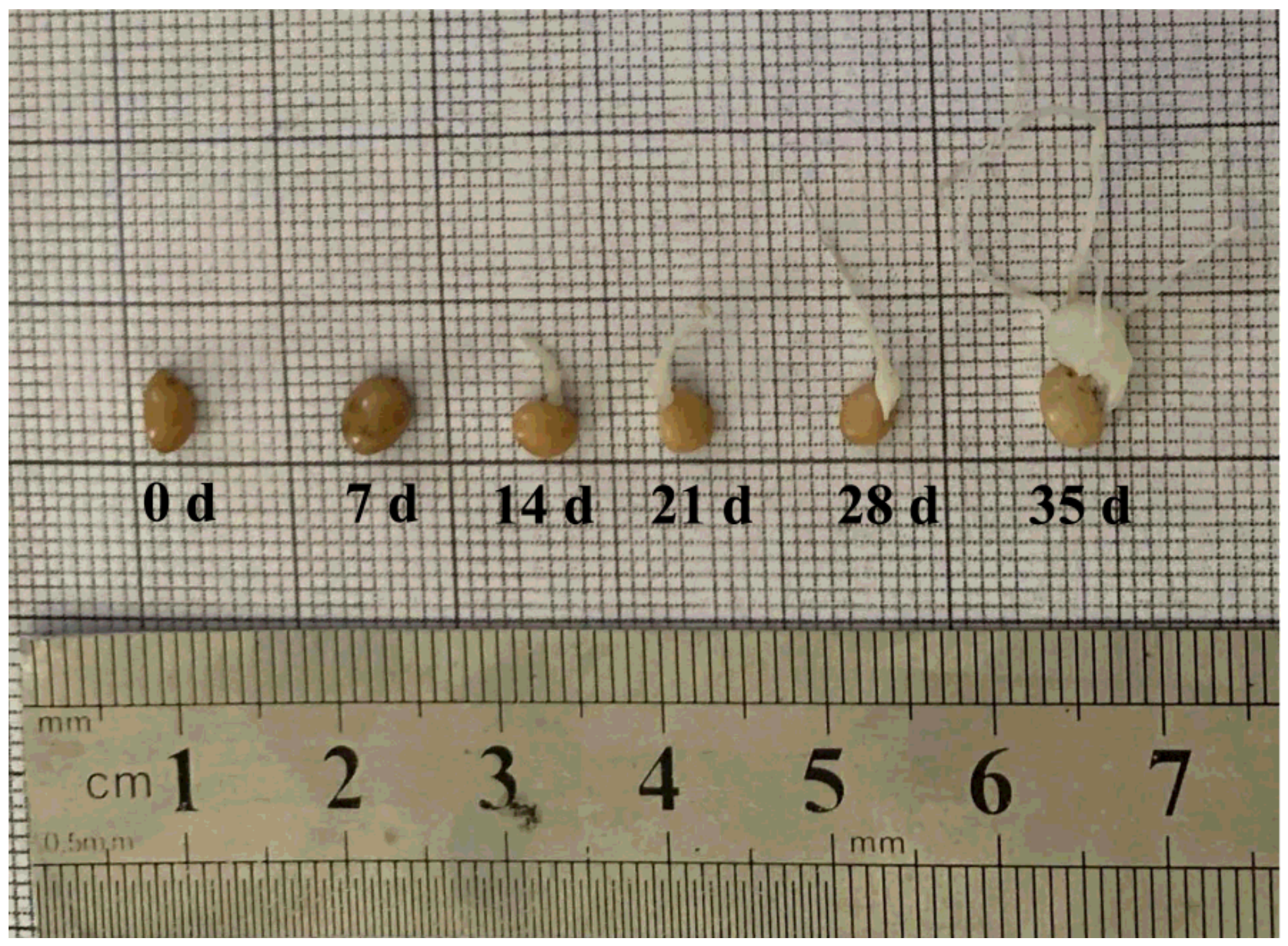

Figure 5

Seeds of P. cyrtonema Hua at different germination stages 


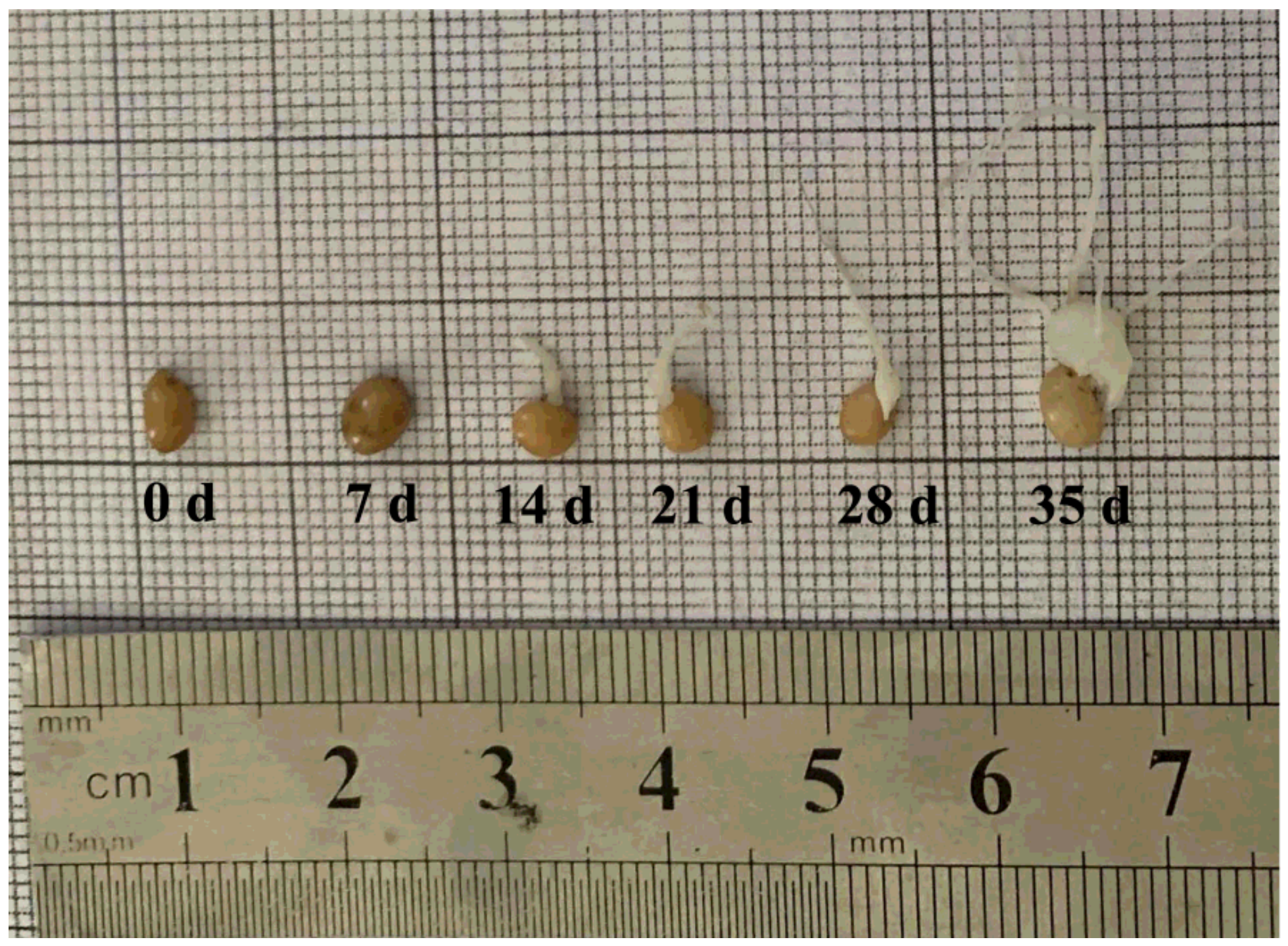

Figure 5

Seeds of P. cyrtonema Hua at different germination stages 


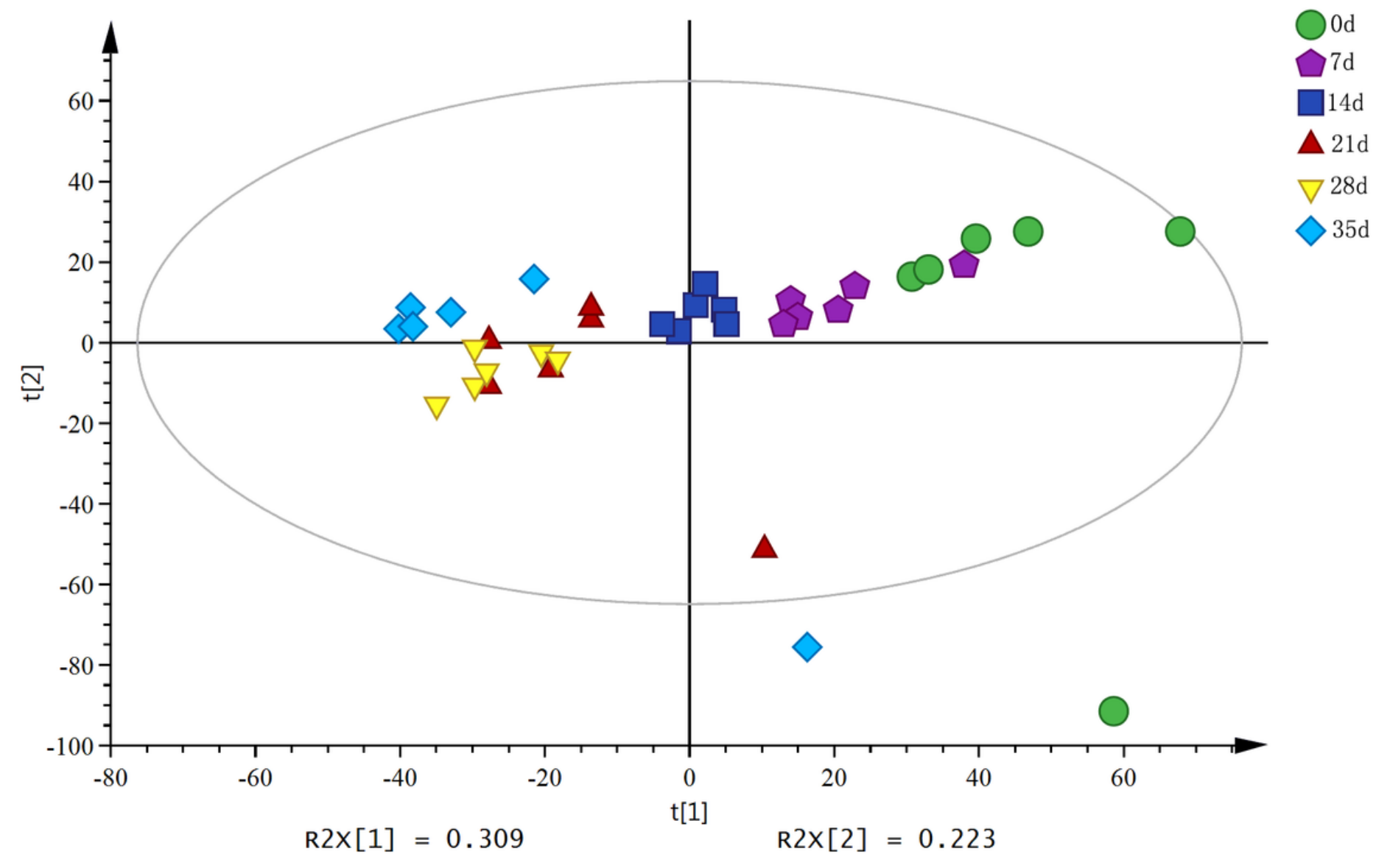

Figure 6

Principal component analysis $₫ P C A \rrbracket$ of metabolites of P. cyrtonema Hua seeds at different germination stages 


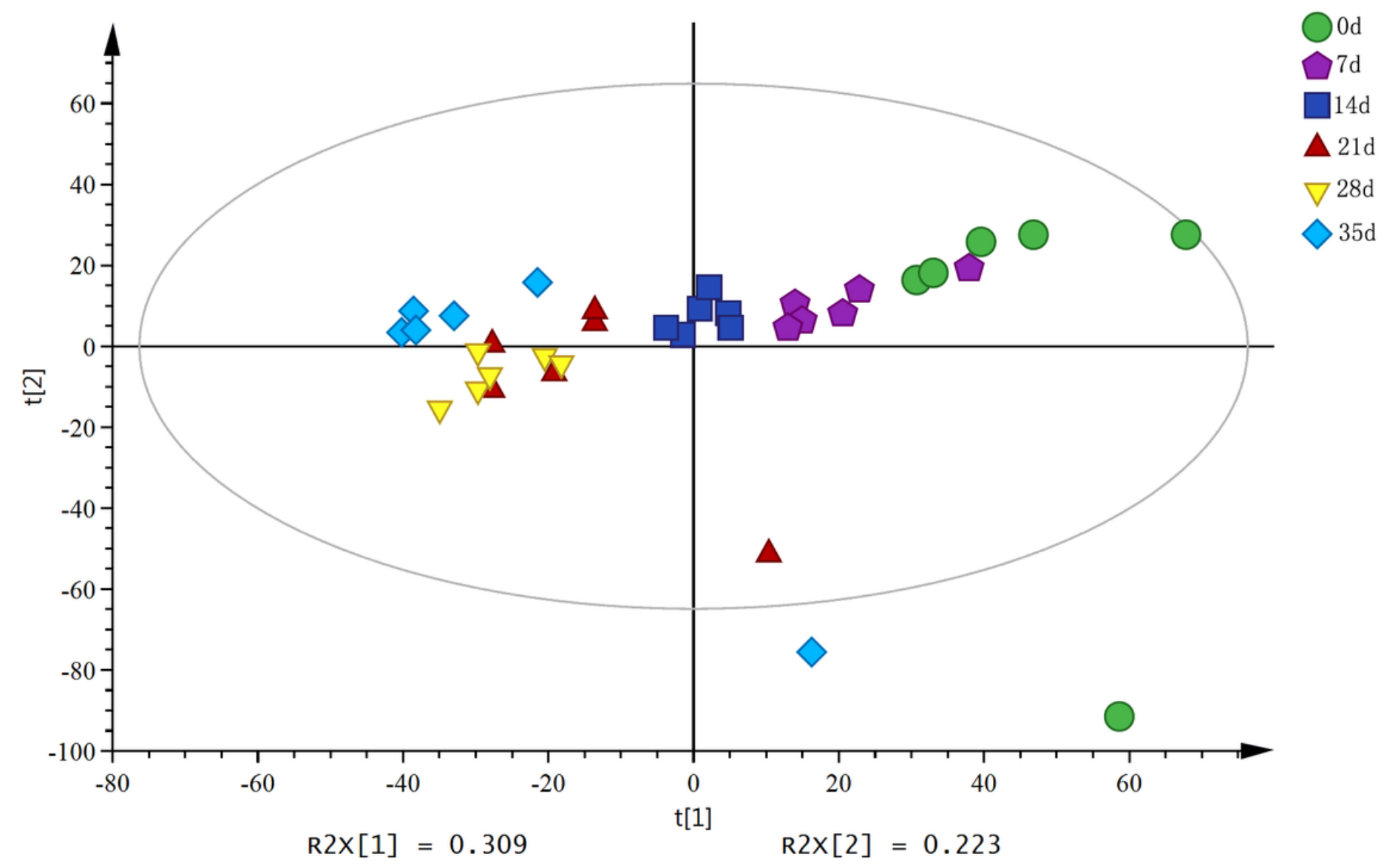

Figure 6

Principal component analysis $₫ P C A \rrbracket$ of metabolites of P. cyrtonema Hua seeds at different germination stages
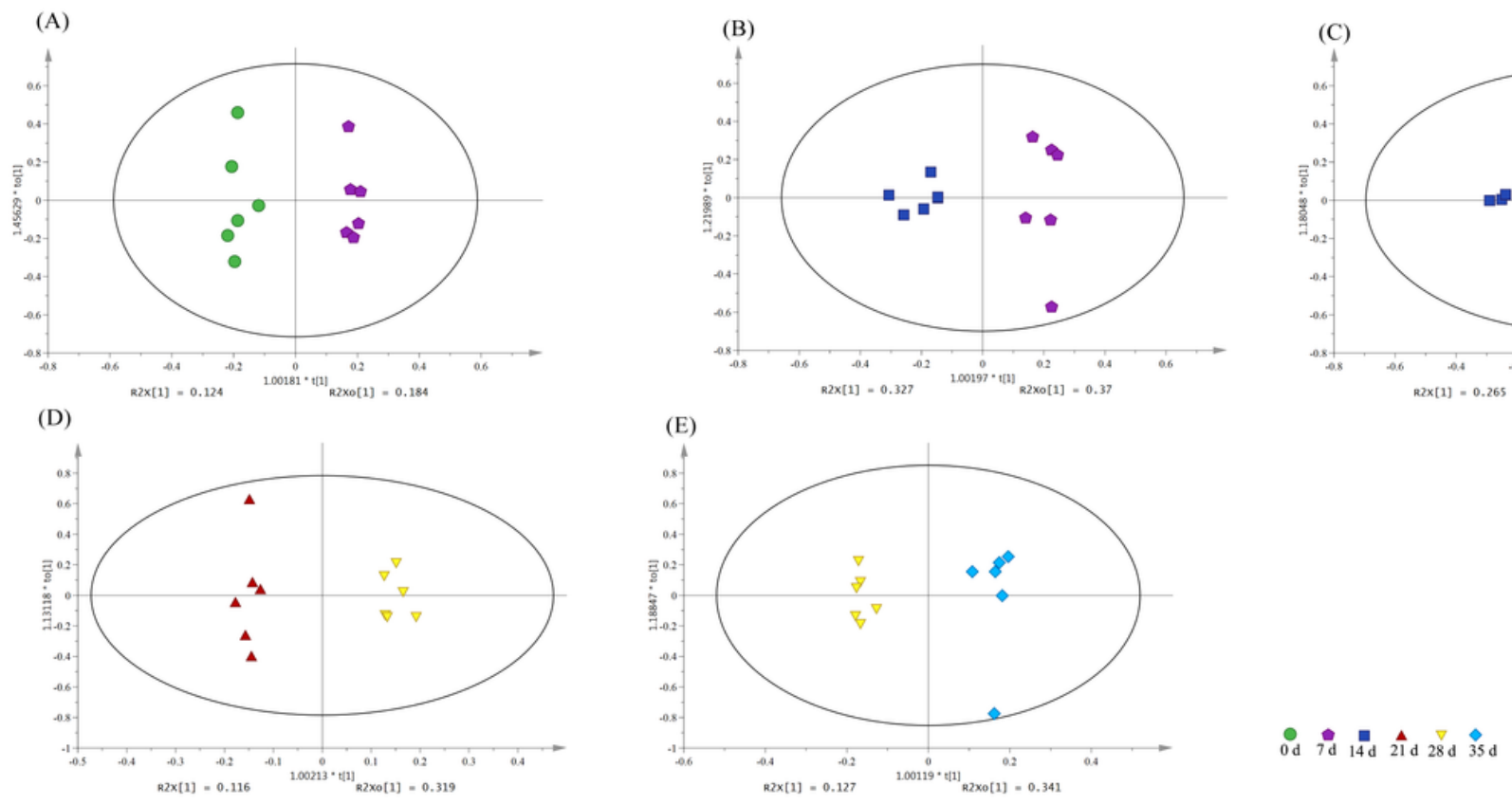

Figure 7 
The OPLS-DA score map of metabolites during seed germination of P. cyrtonema Hua Note: (A), (B), (C), (D) and (E) are the OPLS-DA score maps of metabolites in the germination of P. cyrtonema Hua seeds for 0 d vs. 7 d, 7 d vs. 14 d, 14 d vs. 21 d, 21 d vs. 28 d, and 28 d vs. 35 d, respectively.
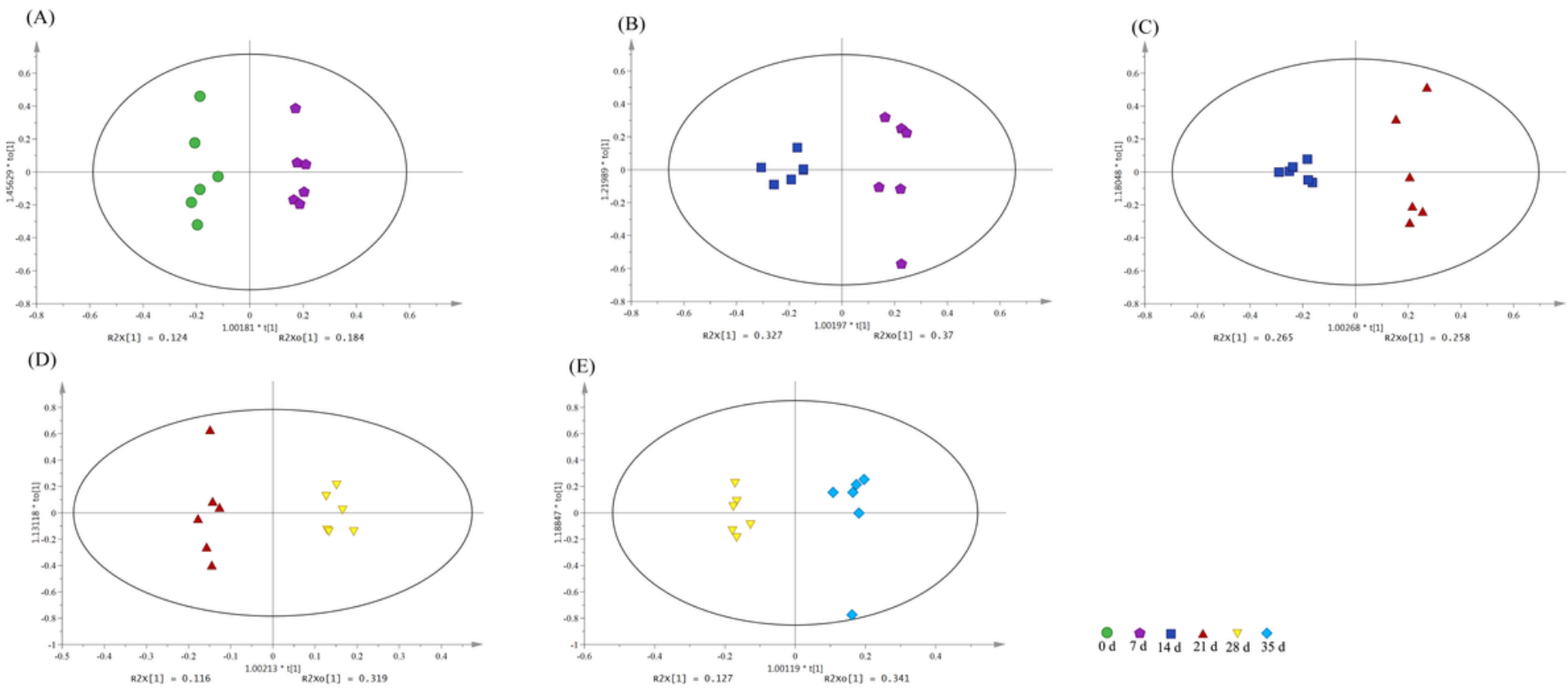

\section{Figure 7}

The OPLS-DA score map of metabolites during seed germination of P. cyrtonema Hua Note: (A), (B), (C), $(D)$ and $(E)$ are the OPLS-DA score maps of metabolites in the germination of P. cyrtonema Hua seeds for $0 \mathrm{~d}$ vs. $7 \mathrm{~d}, 7 \mathrm{~d}$ vs. $14 \mathrm{~d}, 14 \mathrm{~d}$ vs. $21 \mathrm{~d}, 21 \mathrm{~d}$ vs. $28 \mathrm{~d}$, and $28 \mathrm{~d}$ vs. $35 \mathrm{~d}$, respectively. 


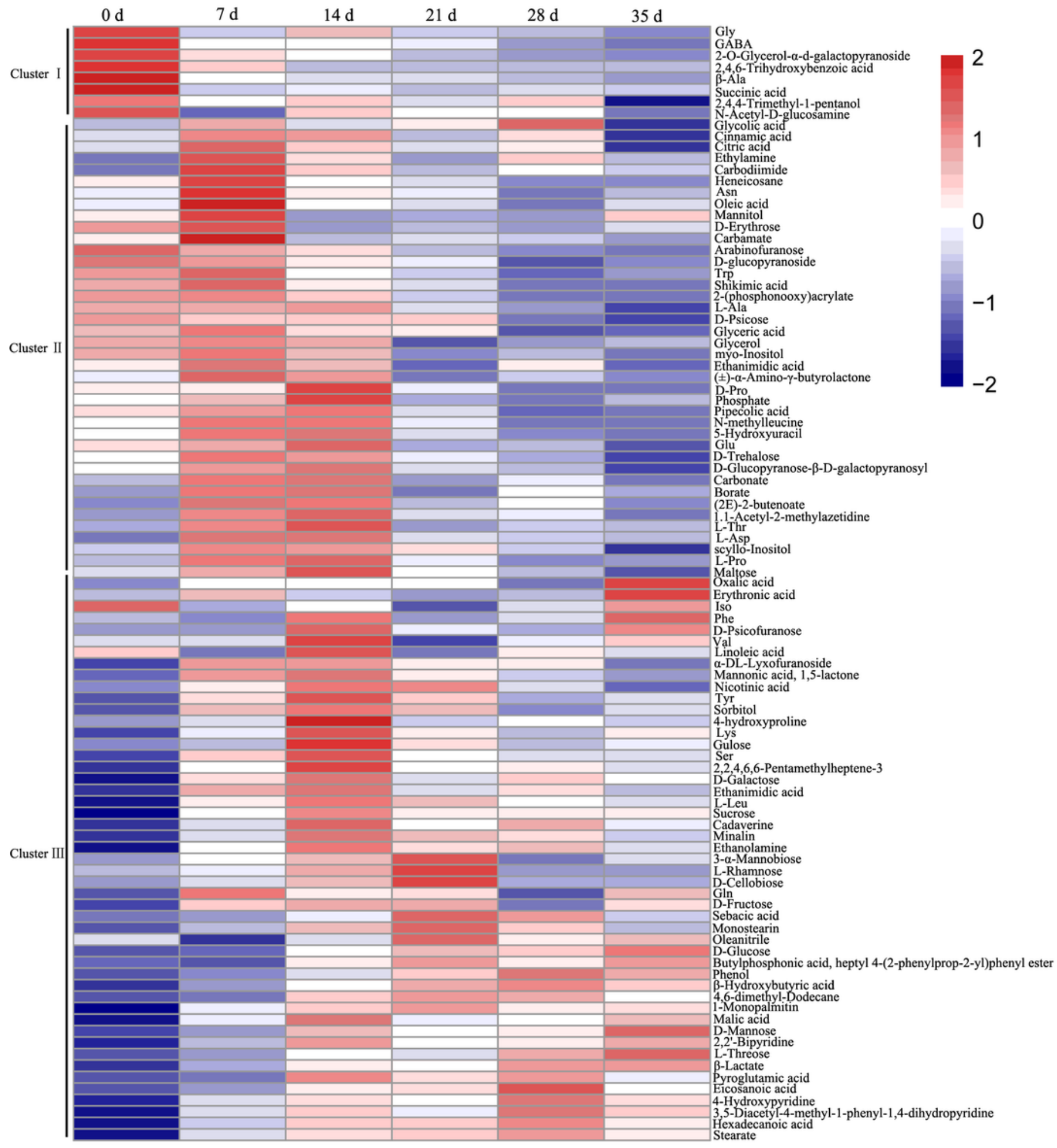

Figure 8

Hierarchical cluster analysis $₫ \mathrm{HCA} \llbracket$ of metabolites during seed germination of P. cyrtonema Hua 


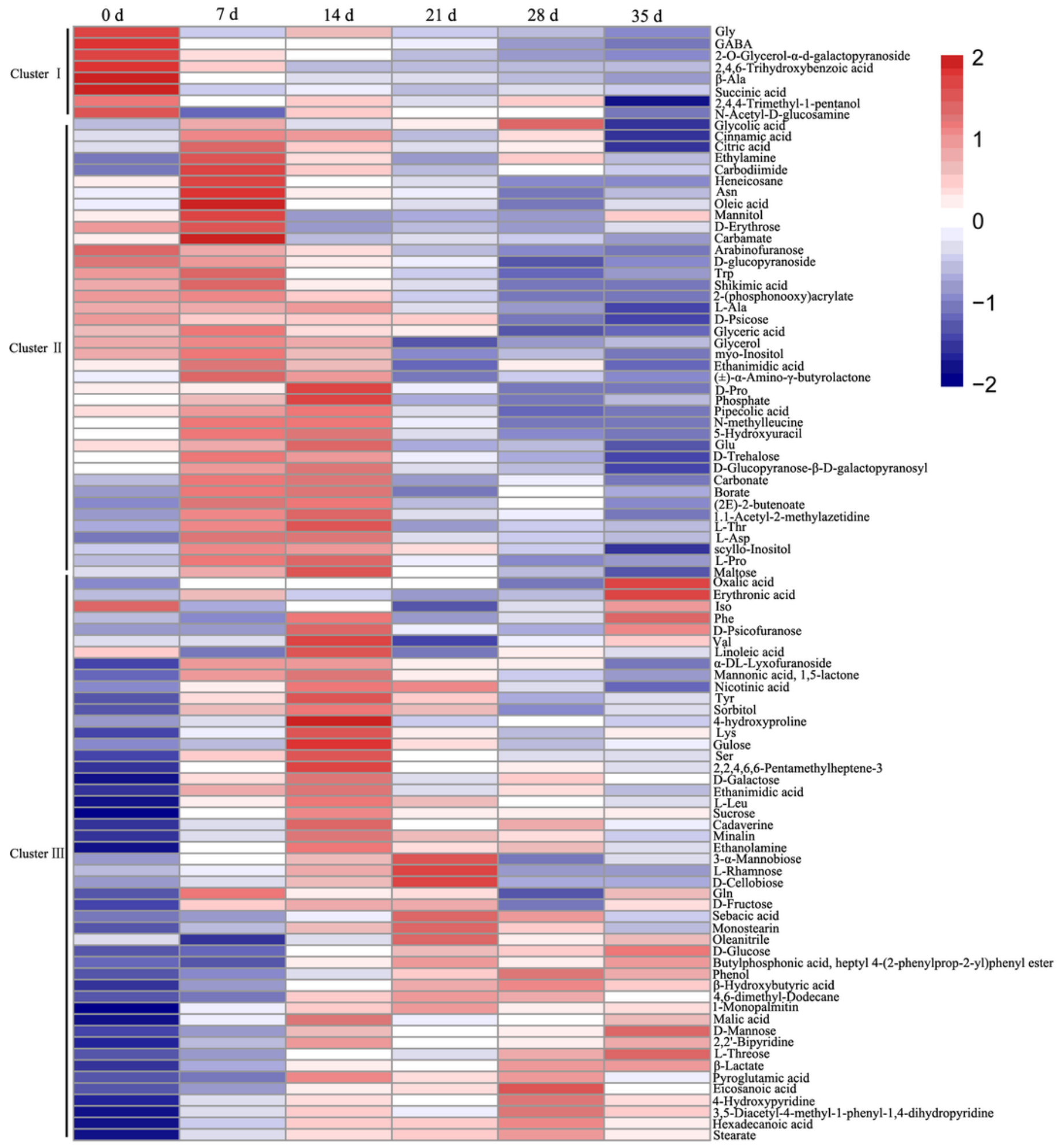

Figure 8

Hierarchical cluster analysis $₫ \mathrm{HCA} \llbracket$ of metabolites during seed germination of P. cyrtonema Hua 

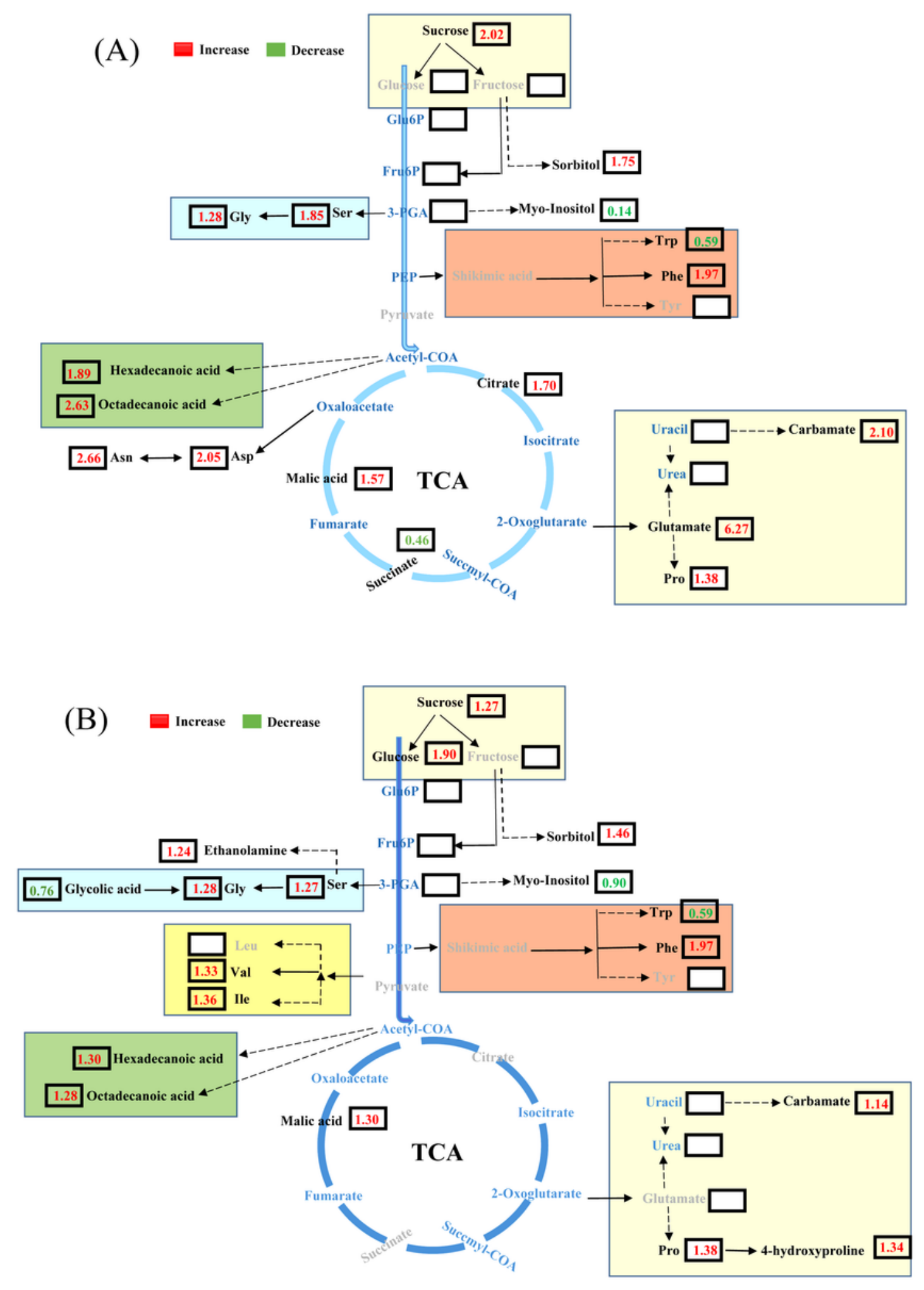

\section{Figure 9}

Metabolic profiling of seed germination of P. cyrtonema Hua Note:(A) and (B) are metabolic profiling of differential metabolites in P. cyrtonema Hua seeds at $7 \mathrm{~d}$ of germination and at $14 \mathrm{~d}$ respectively. The red number represents an increase in metabolite content. The green number represents a decrease in metabolite content. Black font represents differential metabolites, orange font represents non-differential metabolites, blue font represents undiscovered metabolites; Glu 6P, glucose 6-phosphate; Fru6P, fructose- 
6-phosphate; 3-PGA, 3-phosphate glyceraldehyde; PEP, phosphoenolpyruvate; Gly, glycine; Serine; Leu, leucine; Valine; Ile, isoleucine; Trp, tryptophan; Phe, phenylalanine; Tyr, lysine; Pro, proline
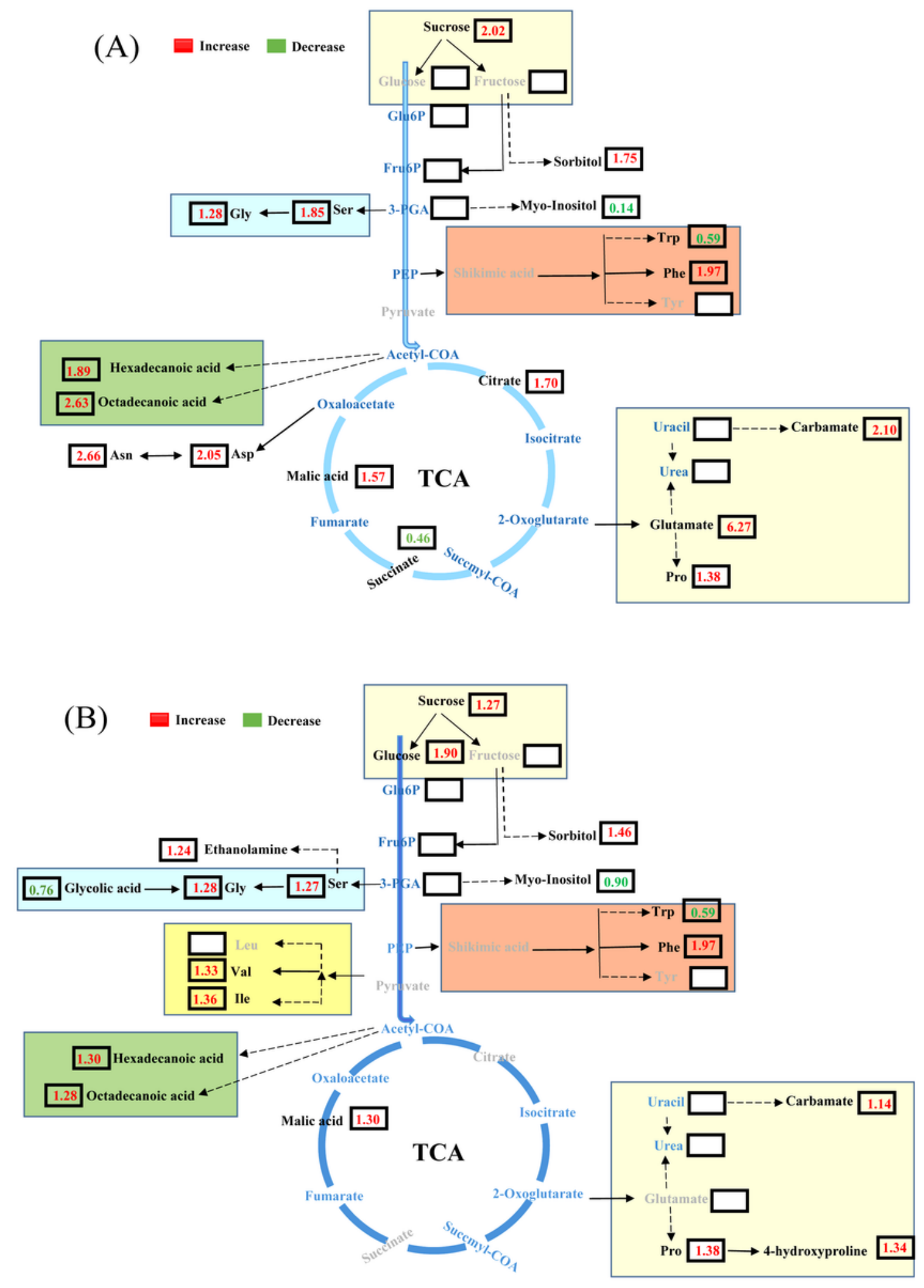

Figure 9

Metabolic profiling of seed germination of P. cyrtonema Hua Note:(A) and (B) are metabolic profiling of differential metabolites in P. cyrtonema Hua seeds at $7 \mathrm{~d}$ of germination and at $14 \mathrm{~d}$ respectively. The red number represents an increase in metabolite content. The green number represents a decrease in 
metabolite content. Black font represents differential metabolites, orange font represents non-differential metabolites, blue font represents undiscovered metabolites; Glu 6P, glucose 6-phosphate; Fru6P, fructose6-phosphate; 3-PGA, 3-phosphate glyceraldehyde; PEP, phosphoenolpyruvate; Gly, glycine; Serine; Leu, leucine; Valine; Ile, isoleucine; Trp, tryptophan; Phe, phenylalanine; Tyr, lysine; Pro, proline

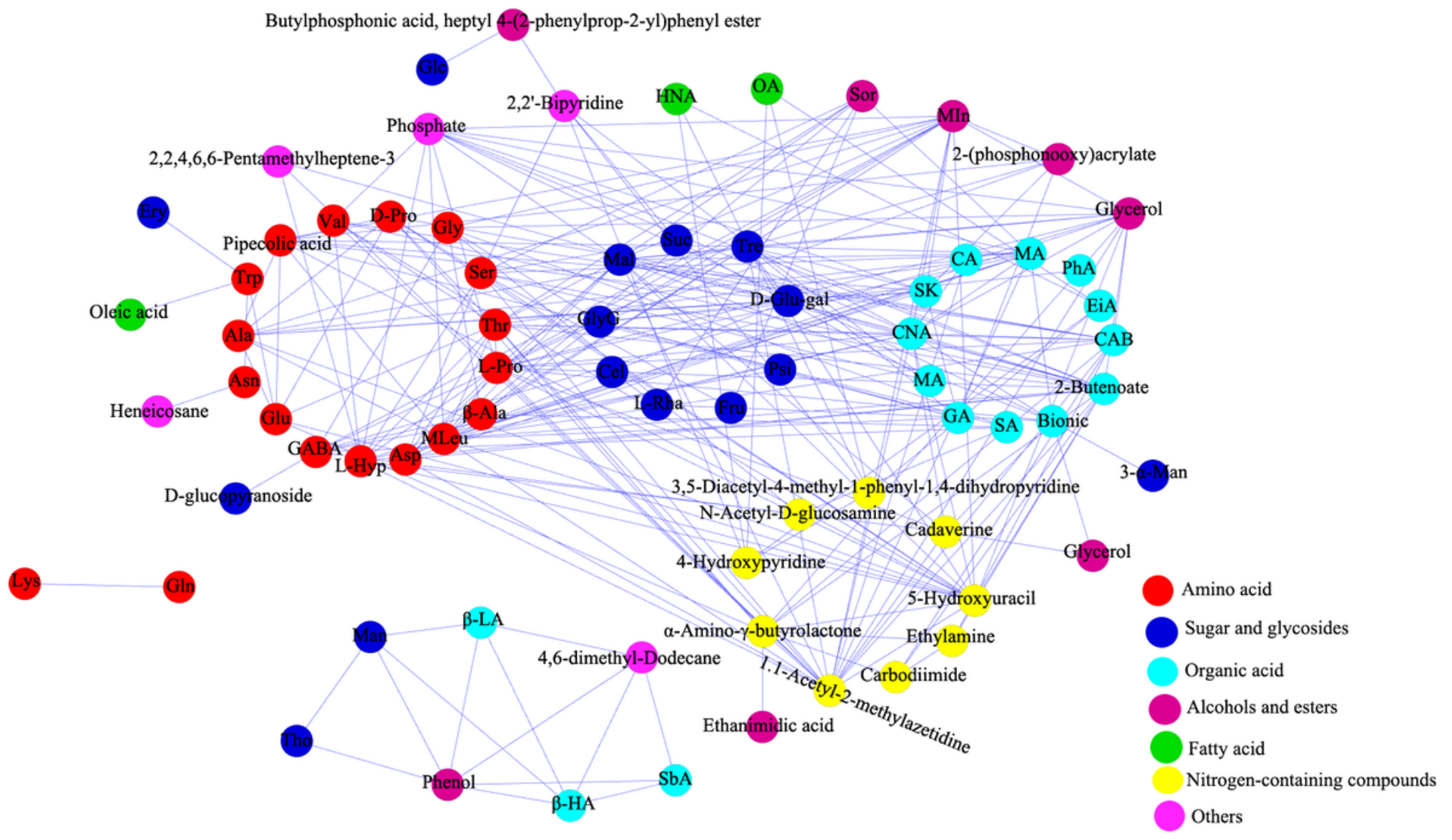

Figure 10

Metabolite network based on significant correlations during seed germination of P. cyrtonema Hua Note: Nodes represent metabolites, and edges represent metabolite relationships. Different node colors indicate metabolites in different pathways. Blue edges show positive correlations 


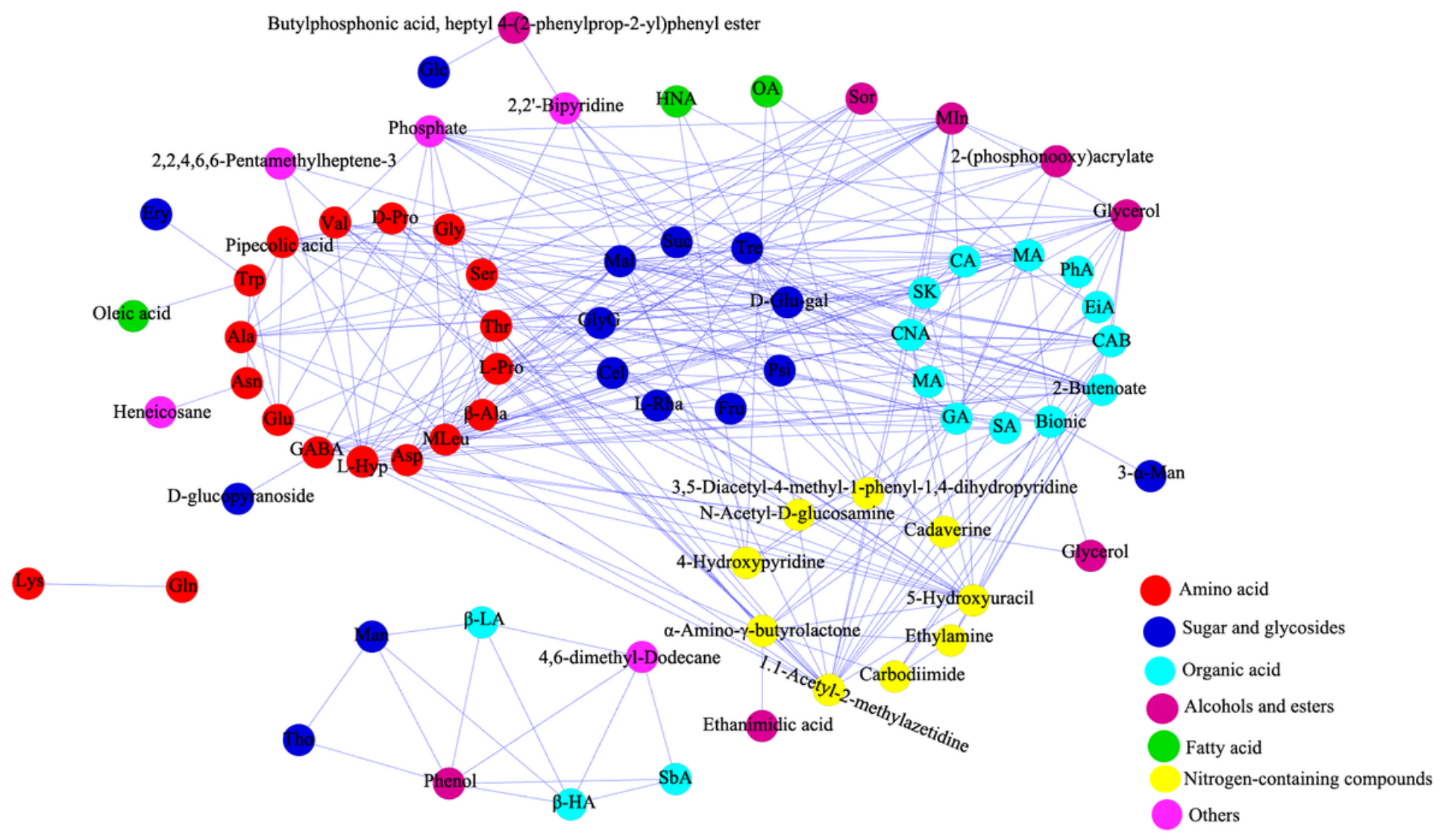

Figure 10

Metabolite network based on significant correlations during seed germination of P. cyrtonema Hua Note: Nodes represent metabolites, and edges represent metabolite relationships. Different node colors indicate metabolites in different pathways. Blue edges show positive correlations

\section{Supplementary Files}

This is a list of supplementary files associated with this preprint. Click to download.

- FigureS1.pdf

- Figures1.pdf

- Figures2.pdf

- Figures2.pdf 